\title{
Stability of Moving Fronts in the Ginzburg-Landau Equation
}

\author{
J. Bricmont ${ }^{1 \star}$, A. Kupiainen ${ }^{2 \star \star}$ \\ ${ }^{1}$ UCL, Physique Theorique, Louvain-la-Neuve, Belgium \\ 2 Rutgers University, Mathematics Department, New Brunswick NJ 08903, USA
}

Received: 13 January 1993/in revised form: 24 March 1993

\begin{abstract}
We use Renormalization Group ideas to study stability of moving fronts in the Ginzburg-Landau equation in one spatial dimension. In particular, we prove stability of the real fronts under complex perturbations. This extends the results of Aronson and Weinberger to situations where the maximum principle is inapplicable and constitutes a step in proving the general marginal stability hypothesis for the Ginzburg-Landau equation.
\end{abstract}

\section{Introduction}

There are very few general approaches to the study of long time existence and asymptotics of solutions of nonlinear parabolic partial differential equations. Typically one has to resort to the use of positivity properties of the linear semigroup, e.g. the use of maximum principle and then use comparison theorems together with compactness arguments to obtain the asymptotics. Such approaches usually work only for equations of special form, e.g. with second order linear part, and positive initial data.

It was noted in $[3,12,13]$, that scaling and renormalization group (RG) concepts that were very successful in statistical mechanics and quantum field theory are also applicable to this study. In $[5,6]$ we have been developing a mathematical RG theory to prove global existence and detailed long time asymptotics for classes of nonlinear parabolic equations. The RG approach does not depend on the applicability of positivity conditions such as the maximum principle. The theory moreover shows how universality emerges in such equations: the long time asymptotics is independent on the initial data and the equation within classes of data and equations. 
In this paper we study one such problem lacking positivity, the stability of moving front solutions in the Ginzburg-Landau equation

$$
\dot{u}=\partial^{2} u+u-|u|^{2} u,
$$

where $u: \mathbf{R} \times \mathbf{R} \rightarrow \mathbf{C}$ is complex, $\partial=\frac{\partial}{\partial x}$ and the dot denotes the time derivative.

There is an extensive study $[1,4]$ of the stability of moving front solutions to (1):

$$
u=r_{c}(x-c t),
$$

where $r_{c}$ is a real (and non-negative) function. The analysis is based on the use of the maximum principle and applies to the case of positive initial data of (1). However, for complex data very little is known, because the maximum principle is no longer applicable. We show in this paper how the RG ideas can be used to prove the stability of the real front solutions (2) under complex perturbations.

Equation (1) has been an important model in the physics literature for the study of velocity selection for propagating patterns $[9,10,2]$. Equation (1) has solutions (2) for a whole range of values of $c$ (see Sect. 2) and, for $c \geqq 2$ the solutions are linearly stable in a space of functions with a prescribed $c$-dependent exponential decay at infinity. A natural question is: which of these moving fronts, if any, is selected for an initial data of compact support (this is also the physically realistic case)? The marginal stability hypothesis [9] states that the marginally stable $c=2$ is the one selected. For real positive initial data this indeed is proven by Aronson and Weinberger $[1,4]$. For complex data there is a large set of complex front solutions $[2,8]$, many of which are linearly stable [8]. An outstanding problem is to prove or disprove the marginal stability hypothesis for complex compactly supported data (for an argument, see [2]). While this is still beyond our methods, the present paper can be considered as a step in this direction.

\section{Results}

We write the Ginzburg-Landau equation in radial and angle variables, $\mathbf{u}=r e^{i \varphi}$ :

$$
\begin{aligned}
& \dot{r}=\partial^{2} r+r\left(1-(\partial \varphi)^{2}\right)-r^{3}, \\
& \dot{\varphi}=\partial^{2} \varphi+2 r^{-1} \partial \varphi \partial r .
\end{aligned}
$$

It is well known that these equations have real, positive, front solutions, i.e. solutions of the form

$$
\varphi=0, \quad r=r_{c}(x-c t) \geqq 0,
$$

such that $r_{c}$ interpolates between a stable and an unstable solution of (1), i.e. $r_{c} \rightarrow+1$ for $x \rightarrow-\infty, r_{c} \rightarrow 0$, for $x \rightarrow+\infty$. Indeed, from (1), we see that $r_{c}$ satisfies

$$
r_{c}^{\prime \prime}+c r_{c}^{\prime}+r_{c}-r_{c}^{3}=0
$$

which, if we reinterpret the variable as "time," can be seen as Newton's equation of motion of a particle of mass one subjected to a friction term $c r_{c}^{\prime}$ and to a force 
deriving from the potential $\frac{r^{2}}{2}-\frac{r^{4}}{4}$, which is an inverted double-well. It is intuitively clear and easily proved that, for $c$ not too small, solutions exists that satisfy the required conditions, i.e. such that $r_{c}$ tends, as "time" goes to $+\infty$, to zero, the stable critical point of the potential, and to one as "time" goes to $-\infty$. For large "time" $u, r_{c}(u)$ will decay exponentially, as is seen from the linearization of (4) at $r=0$. One gets

$$
r_{c}(u) \leqq\left(c_{1}+c_{2} u\right) e^{-\gamma u},
$$

where $\gamma$ is given by $\gamma^{2}-c \gamma+1=0$, i.e.

$$
\gamma_{c}=\frac{1}{2}\left(c-\sqrt{c^{2}-4}\right)
$$

which is real for $c \geqq 2$, in which case $\gamma_{c} \geqq 1$ (actually, one can take $c_{2}=0$ in (5), if $\gamma<1$ ). Thus, the larger the friction, the slower the decay. For $c<2$, the solution "overshoots" the minimum at zero, i.e. $r_{c}$ is no longer positive. Each of the solutions $r_{c}$ with $c \geqq 2$ is stable under real perturbations $(\varphi=0)$ : if we start with initial data $r(x, 0)$, with $r=r_{c}+s$ with $0 \leqq r \leqq 1, s$ decaying faster than $e^{-\gamma_{c} x}$ for $x \rightarrow+\infty$, $r(x, t)$ will converge, as $t \rightarrow+\infty$, to $r_{c}(x-c t)$, see $[1,4]$.

However the solution with $c=2, \gamma_{c}=1$ is more stable than the others in the sense that any initial data $r(x, 0)$ with $0 \leqq r \leqq 1$ which decays faster than $e^{-x}$ as $x \rightarrow+\infty$ (in particular, if $r$ is of compact support) will converge, as $t \rightarrow+\infty$, to $r_{2}(x-2 t)[1,4]$.

Now we consider a complex perturbation of $r_{c}: r(x, 0)=r_{c}+s$ with $\varphi(x, 0) \neq 0$ and $s(x, 0)$ small in a suitable sense. The equations satisfied by $\varphi$ and $s$ are:

$$
\begin{aligned}
\dot{\varphi} & =\partial^{2} \varphi+2\left(\partial r_{c} \partial \varphi+\partial s \partial \varphi\right)\left(r_{0, c}+s\right)^{-1}, \\
\dot{s} & =\partial^{2} s+s\left(1-3 r_{c}^{2}\right)-r_{c}(\partial \varphi)^{2}-s(\partial \varphi)^{2}-3 r_{c} s^{2}-s^{3} .
\end{aligned}
$$

We want to solve these equations for all $t \geqq 1$ with a given initial data $\varphi(x, 1)=\varphi(x), s(x, 1)=s(x)$ (it will be notationally convenient to take the initial time $t=1)$. We will state the main result only in the hardest case $c=2$ and will comment on $c>2$ in the course of the proof. We consider the initial data in the Banach space of $C^{1}$-functions $\varphi, s$ with the norm

$$
\|(\varphi, s)\|=\sup _{x}(1+|x|)^{3+\delta}\left(|\varphi(x)|+\left|\varphi^{\prime}(x)\right|+\left(1+e^{x}\right)\left(|s(x)|+\left|s^{\prime}(x)\right|\right)\right)
$$

and prove the

Theorem. For any $\delta>0$ there exists an $\varepsilon>0$ such that Eqs. $(7,8)$ with $c=2$ and initial data $\varphi(x, 1)=\varphi(x), s(x, 1)=s(x)$ with $\|(\varphi, s)\|<\varepsilon$ have a unique classical solution $\varphi(x, t), s(x, t)$, for all $t \geqq 1$, such that

$$
\begin{aligned}
& |\varphi(x, t)| \leqq t^{-\frac{1}{2}+\delta}\left(1+\frac{|x|}{\sqrt{t}}\right)^{-1-\frac{\delta}{3}}, \\
& |s(x, t)| \leqq t^{-1+\delta}\left(1+e^{u}\right)^{-1}\left(1+\frac{|x|}{t}\right)^{-1-\frac{\delta}{3}} .
\end{aligned}
$$


Remark. We actually solve the integral equations associated to $(7,8)$ (see $(3.14)$, (3.15) below) in a Banach space whose norm is related to (9). This yields more detailed and slightly sharper bounds, as well as bounds on $\partial \varphi(x, t), \partial s(x, t)$. Moreover, it is not difficult to show, using the regularity of the heat kernel and integration by parts that these solutions are actually classical solutions.

Before we go to the proof of the theorem, we want to discuss the result in an informal manner. Since $r_{c}$ is a function of $x-c t$ it is convenient to consider also the equation in the frame of reference of the front: let $u=x-c t$ and $\varphi_{f}(u, t)=\varphi(u+c t, t), s_{f}(u, t)=s(u+c t, t)$; then $\varphi_{f}$ and $s_{f}$ satisfy equations like $(7,8)$, with $c \partial \varphi_{f}$ added to the RHS of (7) and $c \partial s_{f}$ added to the one of (8). Now $r_{c}=r_{c}(u)$ is time-independent.

To understand the expected behaviour of $\varphi_{f}(u, t)$, let us consider the linearised equation around the zero solution:

$$
\dot{\varphi}_{f}=\partial^{2} \varphi_{f}+2 \partial r_{c} \partial \varphi_{f} r_{c}^{-1}+c \partial \varphi_{f} .
$$

It is convenient to rewrite this equation as an imaginary time Schrödinger equation: Let

$$
\varphi_{f}(u, t)=e^{-\frac{c}{2} u} r_{c}(u)^{-1} \psi(u, t)
$$

Then $\psi$ satisfies

$$
\dot{\psi}=\partial^{2} \psi-V \psi
$$

with

$$
V=\frac{c^{2}}{4}+\frac{r_{c}^{\prime \prime}}{r_{c}}+c \frac{r_{c}^{\prime}}{r_{c}}=\frac{c^{2}}{4}-1+r_{c}^{2} .
$$

To derive the last equality, we used Eq. (4), satisfied by $r_{c}$. Since $r_{c} \simeq 1$ for $u \rightarrow-\infty, r_{c} \simeq e^{-\gamma u}$ for $u \rightarrow+\infty$, we have $V \simeq c^{2} / 4$ for $u \rightarrow-\infty, V \simeq c^{2} / 4-1$ for $u \rightarrow+\infty$. So, starting with $\psi(u, 0)$ localized around $u=0$, we expect

$$
\psi(u, t) \sim\left\{\begin{array}{ll}
\frac{e^{-\frac{c^{2} t}{4}-\frac{u^{2}}{4 t}}}{\sqrt{t}} & u \rightarrow-\infty \\
\frac{e^{-\left(\frac{c^{2}}{4}-1\right) t-\frac{u^{2}}{4 t}}}{\sqrt{t}} & u \rightarrow+\infty
\end{array} .\right.
$$

Hence,

$$
\varphi_{f}(u, t) \sim\left\{\begin{array}{ll}
\frac{e^{-\frac{c^{2} t}{4}-\frac{u^{2}}{4 t}-\frac{c}{2} u}}{\sqrt{t}} & u \rightarrow-\infty \\
\frac{e^{-\left(\frac{c^{2}}{4}-1\right) t-\frac{u^{2}}{4 t}-\left(\frac{c}{2}-\gamma\right) u}}{\sqrt{t}} & u \rightarrow+\infty
\end{array},\right.
$$


which, using (6) under the form $\frac{c^{2}}{4}-1=\frac{(c-2 \gamma)^{2}}{4}$, can be written as

$$
\varphi_{f}(u, t) \sim \begin{cases}\frac{e^{-\frac{(u+c t)^{2}}{4 t}}}{\sqrt{t}} & u \rightarrow-\infty \\ \frac{e^{-\frac{(u+(c-2 \gamma) t)^{2}}{4 t}}}{\sqrt{t}} & u \rightarrow+\infty\end{cases}
$$

Since $u+c t=x$, the first part of (17) is a diffusive wave stationary in the fixed frame. Since $c-2 \gamma \geqq 0$ (see (6)), the second part vanishes rapidly, as $u \rightarrow+\infty$, except when $c=2, \gamma=1$. There $\varphi$ contains also a diffusive wave which is "carried along" by the front. This is a rough, but basically correct picture. However, we shall see later that a proper treatment of the effect of the potential yields a different power law than $\frac{1}{\sqrt{t}}$ in (17). From now on, we shall concentrate on the most interesting front namely the one with $c=2, \gamma=1$ and write $r$ for $r_{2}$.

Let us consider the linear equation for $s_{f}$ in that case, in the front frame:

$$
\dot{s}_{f}=\partial^{2} s_{f}+2 \partial s_{f}+s_{f}\left(1-3 r^{2}\right) .
$$

Writing $s_{f}=e^{-u} \sigma$, we get

$$
\dot{\sigma}=\partial^{2} \sigma-\tilde{V} \sigma
$$

with $\tilde{V}=3 r^{2}$.

Following the analysis leading to (17), we get

$$
s(u, t) \sim \begin{cases}\frac{e^{-3 t-\frac{u^{2}}{4 t}-u}}{\sqrt{t}}=\frac{e^{-2 t}}{\sqrt{t}} e^{-\frac{(u+2 t)^{2}}{4 t}} & \text { as } u \rightarrow-\infty \\ \frac{e^{-\frac{u^{2}}{4 t}}}{\sqrt{t}} e^{-u} & \text { as } u \rightarrow+\infty\end{cases}
$$

There is a "wave" which is stationary in the front frame, but exponentially decreasing in $u$, while the wave which stays in the fixed frame is suppressed by the factor $e^{-2 t}$. Of course, when we take into account the nonlinear terms in $(7,8)$, we see that a term like $-r(\partial \varphi)^{2}$ will prevent $s$ from decaying exponentially in time, due to the slower decay of $\varphi$, see (17).

\section{The Proof}

3.1. Preliminaries: The RG-setup. The proof of the theorem is based on the Renormalization Group method $[12,5,6]$, which for the purposes of the present work consists of the Picard iteration composed with a scaling. Let us define

$$
\begin{aligned}
\varphi_{n}(x, t) & \equiv \varphi\left(L^{n} x, L^{2 n} t\right), \\
s_{n}(x, t) & \equiv s\left(L^{n} x, L^{2 n} t\right),
\end{aligned}
$$


and

$$
\begin{aligned}
& \Phi_{n}(x) \equiv \varphi\left(L^{n} x, L^{2 n}\right)=\varphi_{n}(x, 1), \\
& S_{n}(x) \equiv s\left(L^{n} x, L^{2 n}\right)=S_{n}(x, 1) .
\end{aligned}
$$

We shall prove, inductively in $n$, bounds on $\varphi_{n}, s_{n}$ for the finite time interval $\left[1, L^{2}\right]$, which will imply (2.10). This is done by controlling the "RG-map" $\mathscr{R}_{n}$, which relates the $\Phi_{n}, S_{n}$ to $\Phi_{n+1}, S_{n+1}$ :

$$
\mathscr{R}_{n}\left(\Phi_{n}, S_{n}\right)=\left(\Phi_{n+1}, S_{n+1}\right) \text {. }
$$

$\mathscr{R}_{n}$ is constructed by solving a scaled version of the original equation $(2.7,2.8)$ for a finite time. For this, it will be more convenient to work in the variable $u$ relative to the moving frame of the front, which under the scaling (1), (2) becomes

$$
u \equiv x-2 L^{n} t
$$

$(c=2$ here) so that, if we define,

$$
\begin{gathered}
\varphi_{f n}(u, t) \equiv \varphi_{n}\left(u+2 L^{n} t, t\right), \\
s_{f n}(u, t) \equiv s_{n}\left(u+2 L^{n} t, t\right),
\end{gathered}
$$

we have

$$
\begin{gathered}
\varphi_{f n}(u, t)=\varphi_{f}\left(L^{n} u, L^{2 n} t\right), \\
s_{f n}(u, t)=s_{f}\left(L^{n} u, L^{2 n} t\right) .
\end{gathered}
$$

We will from now on drop the subscript $f$, and use the variables $x$ and $u$ to distinguish the frame we are in. $\varphi_{n}(u, t), s_{n}(u, t)$ solve the equations

$$
\begin{aligned}
\dot{\varphi}_{n}= & \partial^{2} \varphi_{n}+2 L^{n} \partial \varphi_{n}+2 L^{n} \partial \varphi_{n}\left(\frac{q_{n}+L^{-n} \partial s_{n} / r_{n}}{1+s_{n} / r_{n}}\right) \\
\dot{s}_{n} \equiv & \partial^{2} s_{n}+2 L^{n} \partial s_{n}+L^{2 n} s_{n}\left(1-3 r_{n}^{2}\right)-r_{n}\left(\partial \varphi_{n}\right)^{2} \\
& -s_{n}\left(\partial \varphi_{n}\right)^{2}-3 r_{n} L^{2 n} s_{n}^{2}-L^{2 n} s_{n}^{3}
\end{aligned}
$$

where $r_{n}(u) \equiv r\left(L^{n}\left(x-2 L^{n} t\right)\right)=r\left(L^{n} u\right)$ and $q_{n}(u) \equiv \frac{r^{\prime}\left(L^{n} u\right)}{r\left(L^{n} u\right)}$.

The RG map (5) is now defined by constructing a solution to $(11,12)$ on the time interval $\left[1, L^{2}\right]$ with initial data $\varphi_{n}(u, 1)=\varphi(u), s_{n}(u, 1)=s(u)$ in a suitable Banach space,

$$
\mathscr{R}_{n}(\varphi, s)(\cdot)=\left(\varphi_{n}\left(L \cdot, L^{2}\right), s_{n}\left(L \cdot, L^{2}\right)\right) .
$$

One then wants to show that $\mathscr{R}_{n}$ maps the space into another one so as to be able to iterate (5).

$\mathscr{R}_{n}$ is studied by rewriting $(11,12)$ as integral equations:

$$
\begin{aligned}
\varphi_{n}(u, t) & =\int d v R_{n}^{t-1}(u, v) \varphi_{n}(v, 1)+\int_{0}^{t-1} d \tau \int d v R_{n}^{\tau}(u, v) M(v, t-\tau) \\
& \equiv\left(R_{n}^{t-1} \varphi_{n}(\cdot, 1)\right)(u)+\mathscr{M}_{n}\left(\varphi_{n}, s_{n}\right)(u, t),
\end{aligned}
$$




$$
\begin{aligned}
s_{n}(u, t) & =\int d v Q_{n}^{t-1}(u, v) s_{n}(v, 1)+\int_{0}^{t-1} d \tau \int d v Q_{n}^{\tau}(u, v) N(v, t-\tau) \\
& \equiv\left(Q_{n}^{t-1} s_{n}(\cdot, 1)\right)(u)+\mathscr{N}_{n}\left(\varphi_{n}, s_{n}\right)(u, t),
\end{aligned}
$$

where $R_{n}^{\tau}, Q_{n}^{\tau}$ solve the linearised version of $(11,12)$ :

$$
\begin{aligned}
\dot{\varphi}_{n} & =\partial^{2} \varphi_{n}+2 L^{n}\left(1+q_{n}\right) \partial \varphi_{n}, \\
\dot{s}_{n} & =\partial^{2} s_{n}+2 L^{n} \partial s_{n}+L^{2 n} s_{n}\left(1-3 r_{n}^{2}\right) .
\end{aligned}
$$

Explicitly, see (2.12), (2.13), (2.19),

$$
\begin{aligned}
& R_{n}^{\tau}(u, v) \equiv \exp \left(f_{n}(u)-f_{n}(v)\right)\left(\exp -\tau H_{n}\right)(u, v), \\
& Q_{n}^{\tau}(u, v) \equiv \exp \left(-L^{n} u+L^{n} v\right)\left(\exp -\tau \tilde{H}_{n}\right)(u, v),
\end{aligned}
$$

where,

$$
f_{n}(u) \equiv f\left(L^{n} u\right), \quad f(u)=-u-\log r(u)
$$

and

$$
\begin{gathered}
u=x-2 L^{n} t, \\
v=y-2 L^{n}, \\
t=\tau+1, \\
H_{n}=-\partial^{2}+L^{2 n} r_{0}^{2}\left(L^{n} \cdot\right), \\
\tilde{H}_{n}=-\partial^{2}+3 L^{2 n} r_{0}^{2}\left(L^{n} \cdot\right) .
\end{gathered}
$$

$M, N$ collect the nonlinear terms in $(11,12)$ :

$$
\begin{aligned}
& M=2 L^{n} \partial \varphi_{n}\left(\frac{q_{n}+L^{-n} \partial s_{n} / r_{n}}{1+s_{n} / r_{n}}-q_{n}\right), \\
& N=-r_{n}\left(\partial \varphi_{n}\right)^{2}-s_{n}\left(\partial \varphi_{n}\right)^{2}-3 r_{n} L^{2 n} s_{n}^{2}-L^{2 n} s_{n}^{3} .
\end{aligned}
$$

Since the nonlinear terms involve $s_{n}, \partial s_{n}, \varphi_{n}, \partial \varphi_{n}$, we shall solve Eqs. $(14,15)$ in a Banach space $\mathscr{B}_{n}$ defined by a weighted $L^{\infty}$ norm on $\varphi, s$ and their first derivative, with $n$-dependent weights:

$$
\|(\varphi, s)\|^{(n)}=\sup _{t \in\left[1, L^{2}\right]}\|\varphi(\cdot, t)\|_{1, t}^{(n)}+\sup _{t \in\left[1, L^{2}\right]}\|s(\cdot, t)\|_{2, t}^{(n)}
$$

with

$$
\begin{aligned}
& \|g\|_{1, t}^{(n)}=\left\|\frac{g}{h_{n, t}}\right\|_{\infty}+\left\|\frac{g^{\prime}}{\tilde{h}_{n, t}}\right\|_{\infty}, \\
& \|g\|_{2, t}^{(n)}=\left\|\frac{g}{w_{n, t}}\right\|_{\infty}+\left\|\frac{g^{\prime}}{\tilde{w}_{n, t}}\right\|_{\infty},
\end{aligned}
$$

and where the weights are chosen to reflect the decay rates of $\varphi, s$ :

$$
\begin{aligned}
h_{n, t}(u) & =\left\{\begin{array}{ll}
(1+|x|)^{-1-\delta} & u<0 \\
(1+u)^{-2-\delta}\left(1+L^{n} u\right)^{-1}\left(1+2 L^{n} t\right)^{-1-\delta} & u \geqq 0
\end{array}\right\}, \\
\tilde{h}_{n, t} & =L^{n(1+\delta)} l_{n} h_{n, t}
\end{aligned}
$$


with

$$
l_{n}(u)=L^{-n\left(\frac{1}{4}-\delta\right)}+\left(1+L^{n}|u|\right)^{-\frac{1}{4}}
$$

and

$$
\begin{aligned}
w_{n, t}(u) & =\left\{\begin{array}{ll}
\left(L^{n}+|x|\right)^{-1-\delta} & u<0 \\
(1+u)^{-2-\delta} e^{-L^{n} u}\left(L^{n}+2 L^{n} t\right)^{-1-\delta} & u \geqq 0
\end{array}\right\}, \\
\tilde{w}_{n, t} & =L^{n(1+\delta)} w_{n, t},
\end{aligned}
$$

where $u$ and $x$ are related by (21), i.e. $x$ is a function of $u$ and $t$ which makes the weights $t$-dependent. For a function $g$ independent of $t$, we define $\|g\|_{1}^{(n)}$ as $\|g\|_{1, t}^{(n)}$ above with $t=1$, and similarly for $\|g\|_{2}^{(n)}$. We write $h_{n}, w_{n}$ for $h_{n, 1}, w_{n, 1}$.

Remark. These complicated weights can be understood as follows: as we saw in (2.17), $\varphi$ contains two diffusive waves, one in the fixed frame, one carried by the front; the first term in $h_{n, t}$ correspond to the wave in the fixed frame, which we assume only to decay (in space) like an integrable power. The second term corresponds to the wave carried by the front, which will decay faster (in time), because the potential in (24) creates a barrier at the origin (of the front frame), so it decays essentially like the solution of the heat equation in $[0, \infty]$. This explains the extra factor $\left(1+2 L^{n} t\right)^{-1-\delta}$ (the role of $\delta$ will be discussed later). However, to get this factor, we need a slightly faster decay (in space) than in the first term, so we put $(1+u)^{-2-\delta}$. The factor $\left(1+L^{n} u\right)^{-1}$ is only due to the change of variable $(2.12)$ leading to the potential equation (recall (2.5)). Finally, for later purposes, we choose the coefficients so that $h_{n, t}(u)$ is continuous. Turning to (34), we expect from $(2.20)$ to have only a wave near the front. This is reflected in the second term of (34), where again the factor $e^{-L^{n_{u}}}$ is related to the change of variables leading to (2.20). For the first term, the linear analysis (2.20) would suggest a much faster decay. However, in (2.9), $s$ is coupled to $\varphi$ by the nonlinear terms and the first part of (34) is produced by this coupling. We will see that, when we take derivatives, $\varphi, s$ are multiplied by $L^{n}$, at least for $u$ (and $\tau$ ) small. This is reflected by the factor multiplying $h_{n, t}$ in (32), and by the less refined factor in (35).

The theorem will be deduced from the

Proposition 1. There exists an $n_{0}>0$ such that for all $n \geqq n_{0}$,

$$
\left\|\mathscr{R}_{n}(\varphi, s)\right\|^{(n+1)} \leqq L^{3 \delta-1}\|(\varphi, s)\|^{(n)}
$$

if $\|(\varphi, s)\|^{(n)} \leqq L^{(3 \delta-1) n}$.

Remark. The $n_{0}$ depends on the $\delta$ in $(31-35)$ ( $\delta$ is small, in particular $\left.<\frac{1}{3}\right)$. It is convenient to express the smallness of the initial data in terms of $n_{0}$, that is, we start the RG-iteration not at $n=0$, but at $n=n_{0}$. This is no loss of generality, since we may scale the equations $(2.7,8)$ by $\varphi(x, t)=\phi\left(L^{-n_{0}} x, L^{-2 n_{0}} t\right)$ and $s$ similarly. Then the norm (2.9) will be bounded by the norm (28). The proposition then implies the theorem (with more detailed estimates on the $x$-dependence), for the discrete times $L^{2 n}$. For the remaining times the bounds below will give the result.

For the proposition, we need to control the linear and the nonlinear parts of $\mathscr{R}_{n}$. For the linear part, we have

Lemma 1. Let $t \in\left[1, L^{2}\right]$. Then

$$
\left\|R_{n}^{t-1} \varphi\right\|_{1, t}^{(n)} \leqq L^{2 n \delta}\|\varphi\|_{1}^{(n)}, \quad\left\|Q_{n}^{t-1} S\right\|_{2, t}^{(n)} \leqq L^{2 n \delta}\|s\|_{2}^{(n)} .
$$


Moreover,

$$
\begin{gathered}
\left\|\left(R_{n}^{L^{2}-1} \varphi\right)(L \cdot)\right\|_{1}^{(n+1)} \leqq L^{-(1-2 \delta)}\|\varphi\|_{1}^{(n)}, \\
\left\|\left(Q_{n}^{L^{2}-1} S\right)(L \cdot)\right\|_{2}^{(n+1)} \leqq L^{-(1-2 \delta)}\|s\|_{2}^{(n)} .
\end{gathered}
$$

Remark. Note how the scaling in the RG produces the contraction lacking in the Picard iteration. The $L^{2 n \delta}$ in (37) is a brute force estimate: actually it could be replaced by $C n$. This divergence arises for very short times. have

For the nonlinear terms we need to use the contraction mapping principle. We

Lemma 2. Let

$$
\|(\varphi, s)\|^{(n)} \leqq 2 L^{-n(1-5 \delta)}
$$

Then

$$
\left\|\left(\mathscr{M}_{n}(\varphi, s), \mathscr{N}_{n}(\varphi, s)\right)\right\|^{(n)} \leqq L^{-n \delta}\|(\varphi, s)\|^{(n)}
$$

and the map $\mathscr{K}_{n}=\left(\mathscr{M}_{n}, \mathscr{N}_{n}\right): \mathscr{B}_{n} \rightarrow \mathscr{B}_{n}$ is a contraction in the ball (40):

$$
\left\|\mathscr{K}_{n}(\varphi, s)-\mathscr{K}_{n}\left(\varphi^{\prime}, s^{\prime}\right)\right\|^{(n)} \leqq \theta\left\|(\varphi, s)-\left(\varphi^{\prime}, s^{\prime}\right)\right\|^{(n)}
$$

for $\theta<1$.

Thus, by (37) and Lemma 2, if we call $\left(\varphi_{n}^{0}, \mathrm{~s}_{n}^{0}\right)$ the linear terms in $(14,15)$, we can solve these equations by the contraction mapping principle applied to a ball of radius $L^{-n(1-5 \delta)}$ around $\left(\varphi_{n}^{0}, s_{n}^{0}\right)$. Indeed, using (36) inductively and (37), one gets $\left\|\left(\varphi_{n}^{0}, s_{n}^{0}\right)\right\|^{(n)} \leqq L^{-n(1-5 \delta)}$. Then (41), (38) and (39) yield (36) i.e. the proposition, and (36), (37) and (41) the Theorem (by redefining $\delta$ ). Hence, we need only to prove Lemmas 1 and 2.

In the course of the proofs, we shall write $C$ or $c$ to denote suitable constants which may vary from place to place (even in the same formula) but do not depend on $L$ or $n$ while $C(L)$ depends on $L$ but not on $n$. $L$ will be fixed, but chosen large enough so that we may repeatedly control constants by writing, e.g.,

$$
C \leqq L^{\delta}
$$

$L$-dependent constants are controlled by

$$
C(L) \leqq L^{\delta n}
$$

since $n \geqq n_{0}$. This explain the proliferation, in the proofs, of powers of $L^{\delta n}$. They are also used to control factors like $C(L) n$. The logic in the choice of $L$ and $n_{0}$ is as follows: given $\delta>0$, small we choose $L$ large enough so that (42) holds for all the $L$-independent constants entering the proofs and then we choose $n_{0}$ so that (43) holds when $n \geqq n_{0}$, for all $L$-dependent constants.

In the proofs, we shall need some properties of the "front" $r$, which follow from an analysis of (2.4). One knows that $r(u) \simeq u e^{-u}$ for $u \rightarrow+\infty$ while $1-r(u) \simeq e^{-c u}$ for $u \rightarrow-\infty$. More precisely, we have for the function $f(u)$ defined in (20),

$$
\begin{gathered}
|f(u)+u| \leqq \lambda e^{c u} \\
\left|f^{\prime}(u)+1\right| \leqq e^{c u}
\end{gathered}
$$


for $u \leqq 0$. Moreover, we can choose the origin of the coordinates so that $\lambda$ in (44) is as small as we wish. This possibility will be used later. Besides, we have

$$
\begin{gathered}
|f(u)+\log (1+u)| \leqq c, \\
\left|f^{\prime}(u)\right| \leqq \frac{c}{1+u}
\end{gathered}
$$

for $u \geqq 0$. Finally, we have (see e.g. $(45,47)$ )

$$
|q(u)|=\left|\frac{r^{\prime}(u)}{r(u)}\right| \leqq c .
$$

3.2. Proof of Lemma 1. We start by proving (38). For this it is enough to show:

$$
\left|\int d v R_{n}^{L^{2}-1}\left(L u^{\prime}, v\right) h_{n}(v)\right| \leqq \frac{1}{2} L^{-(1-2 \delta)} h_{n+1}\left(u^{\prime}\right)
$$

and

$$
\begin{aligned}
& \left|\frac{d}{d u^{\prime}} \int d v R_{n}^{L^{2}-1}\left(L u^{\prime}, v\right) \varphi(v)\right| \\
& \quad \leqq \frac{1}{2} L^{-(1-2 \delta)} L^{(n+1)(1+\delta)} l_{n+1}\left(u^{\prime}\right) h_{n+1}\left(u^{\prime}\right)\left(\left\|\frac{\varphi}{h_{n}}\right\|_{\infty}+\left\|\frac{\varphi^{\prime}}{\tilde{h_{n}}}\right\|_{\infty}\right) .
\end{aligned}
$$

We use primes to denote variables on the scale $n+1$ and unprimed ones for scale $n$. Note that $u^{\prime}=x^{\prime}-2 L^{n+1} t^{\prime}$, so we have $x=L x^{\prime}$ for $u=L u^{\prime}, t=L^{2}$, i.e. $t^{\prime}=1$.

To prove $(49,50)$, we need some properties of $R_{n}^{\tau}$ : we shall use the path space representation,

$$
\left(\exp -\tau H_{n}\right)(u, v)=\int d \mu_{u, v}^{\tau}(\omega) e^{-\int_{0}^{\tau} V_{n}(\omega(s)) d s},
$$

where $d \mu_{u, v}^{\tau}$ is the Brownian bridge going in time $\tau$ from $u$ to $v$, so that $\int d \mu_{u, v}^{\tau}(\omega)=\frac{e^{-(u-v)^{2} / 4 \tau}}{\sqrt{4 \pi \tau}}$ and $V_{n}(\cdot)=L^{2 n} r^{2}\left(L^{n} \cdot\right)$. Thus the potential has a high and sharp barrier around zero which is repulsive on the negative real axis. To get simple estimates we introduce

$$
\varepsilon_{n}=k n L^{-n} \log L,
$$

where $k$ is chosen large so that $V_{n}$ is essentially equal to 0 or to $L^{2 n}$ outside the interval $I_{n}=\left[-\varepsilon_{n},+\varepsilon_{n}\right]$. In Lemma 3 and later, we have bounds where the quantity $L^{-n p}$ enters. Here, $p=\mathcal{O}(k)$ can be taken large by choosing $k$ in (52) large, and the quantities bounded by $L^{-n p}$ will turn out to be negligible. The bounds based on the path space representation (51) will be stated in a series of lemmas, whose proofs will be given in the Appendix.

Lemma 3. For $0 \leqq \tau \leqq L^{2}-1$ and $n \geqq n_{0}$, we have

a) Let $u \geqq 0$. If $v \geqq-\varepsilon_{n}$, then

$$
\exp \left(-\tau H_{n}\right)(u, v) \leqq D_{-2 \varepsilon_{n}}^{\tau}(u, v)+L^{-n p} e^{-\frac{c}{\sqrt{\tau}}(u+v)},
$$


where $D_{a}^{\tau}(u, v)$ is the Dirichlet kernel with barrier at a:

$$
D_{a}^{\tau}(u, v)=H^{\tau}(u-v)-H^{\tau}(u+v-2 a)
$$

for $u, v>a$, with

$$
H^{\tau}(u)=\frac{e^{-\frac{u^{2}}{4 \tau}}}{\sqrt{4 \pi \tau}}
$$

being the heat kernel. Moreover,

$$
\int_{-\infty}^{-\varepsilon_{n}} d v R_{n}^{\tau}(u, v)\left(1+\frac{|v|}{\sqrt{\tau}}\right) \leqq L^{-n p} e^{-c \frac{u}{\sqrt{\tau}}}\left(1+L^{n} u\right)^{-1} .
$$

b) Let $u \leqq 0$. If $v \leqq-\varepsilon_{n}$, then

$$
R_{n}^{\tau}(u, v) \leqq \frac{C}{\sqrt{\tau}} \exp \left(-\frac{\left(u-v+2 L^{n} \tau\right)^{2}}{4 \tau}\right)+L^{-n p} e^{-\frac{c}{\sqrt{\tau}}(|v|+|x| x(x<0))}
$$

and for all $t \in\left[1, L^{2}\right]$,

$$
\begin{aligned}
& \int_{-\varepsilon_{n}}^{\infty} d v R_{n}^{\tau}(u, v) h_{n, t-\tau}(v)\left(1+L^{-n \frac{|u-v|}{\sqrt{\tau}}}\right) \\
& \leqq h_{n, t}(u)\left(L^{-\frac{n}{3}}+L^{\frac{n \delta}{4}} l_{n}(u) \chi\left(\tau \leqq L^{-\frac{n}{2}}\right)\right) e^{-\frac{c}{\sqrt{\tau}}|x| \chi(x<0)} .
\end{aligned}
$$

Remark. Except for (58), these bounds are intuitively obvious: in (53) correction to the Dirichlet kernel comes from excursions to the high potential region, whence the $L^{-n p}$. In (56) such excursions have to take place and in (57) the leading term comes from the heat kernel, since by (21-23),

$$
u-v+2 L^{n} \tau=x-y .
$$

Using Lemma 3 for $u=L u^{\prime}$ and $\tau=L^{2}-1$, we can prove (49). Consider first $u^{\prime} \geqq 0$, and write the integral in (49) as:

$$
\int d v=\int_{-\varepsilon_{n}}^{\infty} d v+\int_{-\infty}^{-\varepsilon_{n}} d v
$$

The second integral is trivial by (56), so consider the first one. Use $(18,53)$, and the bounds

$$
e^{-f_{n}(v)} h_{n}(v) \leqq C L^{-n(1+\delta)}(1+v)^{-2-\delta}
$$

for $v \geqq-\varepsilon_{n}$, and

$$
e^{f_{n}\left(L u^{\prime}\right)} \leqq C\left(1+L^{n+1} u^{\prime}\right)^{-1}
$$

for $u^{\prime} \geqq 0$, which follow from $(31,46,44)$. We see then that all we have to show is

$$
\int_{-\varepsilon_{n}}^{\infty} d v D_{-2 \varepsilon_{n}}^{L^{2}-1}\left(L u^{\prime}, v\right)(1+v)^{-2-\delta} \leqq L^{-2+\frac{\delta}{2}}\left(1+u^{\prime}\right)^{-2-\delta} .
$$

Indeed, the second term in (53) is trivial and, in (63), we write $L^{-2+\frac{\delta}{2}}=L^{-\frac{\delta}{2}} L^{-(1-2 \delta)} L^{-(1+\delta)} ; L^{-(1-2 \delta)}$ occurs in $(49), L^{-(1+\delta)}$ is used, with $L^{-n(1+\delta)}$ in $(61)$, to obtain the factor $\left(1+2 L^{n+1}\right)^{-1-\delta}$ in $h_{n+1}\left(u^{\prime}\right)$ (for $\left.u^{\prime} \geqq 0\right)$ and 
$L^{-\frac{\delta}{2}}$ is used to control constants, as in (42). Equation (63) follows from (54), the definition of $D$ : for $u^{\prime} \geqq k \log L$, with $k$ large, we use simply

$$
D_{-2 \varepsilon_{n}}^{L^{2}-1}\left(L u^{\prime}, v\right) \leqq H^{L^{2}-1}\left(L u^{\prime}-v\right) \leqq \frac{C}{L} e^{-c\left|u^{\prime}-\frac{v}{L}\right|}
$$

and

$$
L^{-1} \int_{-\varepsilon_{n}}^{\infty} d v e^{-c\left|u^{\prime}-\frac{v}{L}\right|}(1+v)^{-2-\delta} \leqq C\left(1+L u^{\prime}\right)^{-2-\delta}
$$

with $\left(1+L u^{\prime}\right)^{-2-\delta} \leqq L^{-2}\left(1+u^{\prime}\right)^{-2-\delta}$ for such $u^{\prime}$.

For $u^{\prime} \leqq k \log L$, we use

$$
D_{a}^{\tau}(u, v)=\left|\int_{u-v}^{u+v-2 a} d z \frac{d}{d z} H^{\tau}(z)\right| \leqq c \tau^{-1}(|v|+|a|)
$$

to get

$$
D_{-2 \varepsilon_{n}}^{L^{2}-1}\left(L u^{\prime}, v\right) \leqq \frac{c}{L^{2}}\left(|v|+\varepsilon_{n}\right)
$$

which gives (63).

For $u^{\prime} \leqq 0$, we use (60) in (49), insert (57) in the second integral and observe that, for $\tau=L^{2}-1, u=L u^{\prime}$, (59) becomes: $L u^{\prime}-v+2 L^{n}\left(L^{2}-1\right)=L x^{\prime}-y$. Using the second inequality in (64) on $H^{L^{2}-1}\left(L x^{\prime}-y\right)$, the first term of the RHS of (57) gives a bound on $\int_{-\infty}^{-\varepsilon_{n}} d v R^{L^{2}-1}(u, v) h_{n}(v)$ of the form

$$
\frac{C}{L} \int_{v \leqq-\varepsilon_{n}} d y e^{-c\left|x^{\prime}-\frac{y}{L}\right|}(1+|y|)^{-1-\delta} \leqq C L^{-1}\left(1+\left|x^{\prime}\right|\right)^{-1-\delta}
$$

which yields a contribution to the RHS of (49) for that part of the integral. The second term in (57) gives another contribution to (49): we get exponential decay for $x^{\prime} \leqq 0$ and, for $x^{\prime} \geqq 0$, we may use part of $L^{-n p}$ to get the factor $\left(1+x^{\prime}\right)^{-1-\delta}$ in $h_{n+1}\left(u^{\prime}\right)$, since $x^{\prime} \leqq 2 L^{n+1}$ for $u^{\prime} \leqq 0$.

Finally, for $\int_{v>-\varepsilon_{n}} d v$, we use (58), where only the term with $L^{-\frac{n}{3}}$ in the RHS contributes, since $t=L^{2}, \tau=L^{2}-1$. Moreover, for $u=L u^{\prime}$ (of any sign),

$$
h_{n, L^{2}}(u) \leqq h_{n+1}\left(u^{\prime}\right)
$$

We use, as in (43), the factor $L^{-\frac{n}{3}}$ in (58) to bound constants, and to give $L^{-(1-2 \delta)}$ in (49).

Now, we prove (50). Let us consider first $u^{\prime} \geqq 0$. We use the following formula, valid for any continuous, piecewise differentiable function $g$ with $\|g\|_{\infty}+\left\|g^{\prime}\right\|_{\infty}<\infty$

$$
\begin{aligned}
\frac{d}{d u}\left(R_{n}^{\tau} g\right)(u) & =\frac{d}{d u} \int d v R_{n}^{\tau}(u, v) g(v) \\
& =L^{n} f^{\prime}\left(L^{n} u\right) \int d v R_{n}^{\tau}(u, v) g(v)
\end{aligned}
$$




$$
\begin{aligned}
& -\int d v e^{f_{n}(u)-f_{n}(v)} \int d \mu_{u, v}^{\tau}(\omega)\left(e^{-\int_{0}^{\tau} V_{n}(\omega(s)) d s} L^{3 n}\right. \\
& \left.\times \int_{0}^{\tau} V^{\prime}\left(L^{n} \omega(s)\right) d s\right) g(v) \\
& -L^{n} \int d v R_{n}^{\tau}(u, v) f^{\prime}\left(L^{n} v\right) g(v) \\
& +\int d v R_{n}^{\tau}(u, v) g^{\prime}(v) d v .
\end{aligned}
$$

To prove (70), use the definition of $R_{n}^{\tau}(18)$, the path space formula (51) and the identity, coming from the translation invariance of $d \mu_{u, v}^{\tau}$ :

$$
\frac{d}{d a} \int d \mu_{u+a, v+a}^{\tau}(\omega) e^{-\int_{0}^{\tau} V_{n}(\omega(s)-a) d s}=0
$$

which implies

$$
\left(\frac{d}{d u}+\frac{d}{d v}\right)\left(e^{-\tau H_{n}}\right)(u, v)=-\int d \mu_{u, v}^{\tau}(\omega)\left(e^{-\int_{0}^{\tau} V_{n}(\omega(s)) d s} L^{3 n} \int_{0}^{\tau} V^{\prime}\left(L^{n} \omega(s)\right) d s\right) .
$$

Integration by parts of the $d / d v$ gives the last two terms of (70).

Consider each term of (70), with $g=\varphi, \tau=L^{2}-1$ and $u=L u^{\prime}(\geqq 0)$ so that $\frac{d}{d u^{\prime}}=L \frac{d}{d u}$. We shall use the fact that in $(50)$, we have a factor $L^{(n+1)(1+\delta)} l_{n+1}\left(u^{\prime}\right)$ while all the terms of (70), except the last one, involve an integration with $\varphi(v)$ instead of its derivative.

For the first term in (70), we use (47), which yields

$$
L^{n+1}\left|f^{\prime}\left(L^{n+1} u^{\prime}\right)\right| \leqq \frac{L^{n+1}}{1+L^{n+1} u^{\prime}} \leqq L^{n+1} l_{n+1}\left(u^{\prime}\right) .
$$

This, combined with (49), gives a contribution to the RHS of (50).

For the last two terms of (70), it is enough to prove that:

$$
\int d v R_{n}^{L^{2}-1}\left(L u^{\prime}, v\right)\left(1+L^{n}|v|\right)^{-\frac{1}{4}} h_{n}(v) \leqq C L^{-\frac{n}{4}} h_{n+1}\left(u^{\prime}\right)
$$

for $u^{\prime} \geqq 0$. Indeed, as in (73), $\left|f^{\prime}\left(L^{n} v\right)\right| \leqq\left(1+L^{n}|v|\right)^{-\frac{1}{4}}$ and we can bound $\varphi^{\prime}$ in terms of $l_{n} h_{n}$. We use (49) for the first term in $l_{n}$. Then, we write $L^{-\frac{n}{4}} \leqq L^{-n \delta} l_{n+1}$. The factor $C$ in (74) and $L^{-(1-2 \delta)}$ in (50) can be controlled using $L^{-n \delta}$ here and (43). To prove (74), we follow the proof of (49) for $u^{\prime} \geqq 0$ and use, instead of (63),

$$
\int_{-\varepsilon_{n}}^{\infty} d v D_{-2 \varepsilon_{n}}^{L^{2}-1}\left(L u^{\prime}, v\right)(1+v)^{-2-\delta}\left(1+L^{n}|v|\right)^{-\frac{1}{4}} \leqq C L^{-\frac{n}{4}}\left(1+u^{\prime}\right)^{-2-\delta},
$$

which is easy to prove, using (63) for $v \geqq 1$ and bounding $D_{-2 \varepsilon_{n}}^{L^{2}-1}\left(L u^{\prime}, v\right)(1+v)^{-2-\delta}$ by $C e^{-c u^{\prime}}$ for $v \leqq 1$.

For the second term in (70), it is enough to show:

$$
\begin{aligned}
L \int d v e^{f_{n}\left(L u^{\prime}\right)-f_{n}(v)} k_{n}\left(L u^{\prime}, v, L^{2}-1\right) h_{n}(v) & \leqq C(L) L^{\frac{2 n}{3}} h_{n+1}\left(u^{\prime}\right) \\
& \leqq L^{-n \delta} L^{(n+1)(1+\delta)} l_{n+1}\left(u^{\prime}\right) h_{n+1}\left(u^{\prime}\right),
\end{aligned}
$$


where the second inequality follows from $(33,43)$, and we used the notation

$$
k_{n}(u, v, \tau)=\int d \mu_{u, v}^{\tau}(\omega)\left(e^{-\int_{0}^{\tau} V(\omega(s)) d s} L^{3 n} \int_{0}^{\tau} \mid V^{\prime}\left(L^{n}(\omega(s)) \mid d s\right) .\right.
$$

The first inequality of (76) follows from parts a and b of the following lemma, for $\tau=L^{2}-1$, and $(61,62)$.

Lemma 4. With the notation (77), we have, for $0 \leqq \tau \leqq L^{2}-1$ and $n \geqq n_{0}$ :

a) for $u \geqq 0$,

$$
\int_{-\varepsilon_{n}}^{\infty} d v k_{n}(u, v, \tau) \leqq e^{-\frac{c}{L} u}\left(L^{\frac{2 n}{3}}+L^{n\left(1+\frac{\delta}{2}\right)} l_{n}(u) \chi\left(\tau \leqq L^{-\frac{n}{2}}\right)\right),
$$

b) for $u \geqq 0$,

$$
\int_{-\infty}^{-\varepsilon_{n}} d v e^{-f_{n}(v)} k_{n}(u, v, \tau) \leqq L^{-n p} e^{-\frac{c}{\sqrt{\tau}} u}\left(1+L^{n} u\right)^{-1},
$$

c) for $u \leqq 0$,

$$
\begin{gathered}
\int_{-\varepsilon_{n}}^{\infty} d v \exp \left(f_{n}(u)-f_{n}(v)\right) k_{n}(u, v, \tau) h_{n, t-\tau}(v) \\
\leqq \\
h_{n, t}(u)\left(L^{n\left(\frac{2}{3}+\frac{\delta}{4}\right)}+L^{n\left(1+\frac{\delta}{2}\right)} l_{n}(u) \chi\left(\tau \leqq L^{-\frac{n}{2}}\right)\right),
\end{gathered}
$$

d) for $u \leqq 0$,

$$
\int_{-\infty}^{-\varepsilon_{n}} d v \exp \left(\dot{f}_{n}(u)-f_{n}(v)\right) k_{n}(u, v, \tau) \leqq L^{-n p} e^{-\frac{c}{\sqrt{\tau}}|x| x(x<0)} .
$$

Now we prove (50) for $u^{\prime} \leqq 0$. We use a different formula for $\frac{d}{d u}\left(R_{n}^{\tau} g\right)(u)$. Write $R_{n}^{\tau}(u, v)=\frac{1}{\sqrt{4 \pi \tau}} e^{f_{n}(u)+L^{n_{u}}-\left(f_{n}(v)+L^{n_{v}}\right)-\left(u-v+2 L^{n^{n}} \tau\right)^{2} / 4 \tau}\left\langle e^{-\int_{0}^{\tau} V(\omega(s)) d s}\right\rangle_{u, v}^{\tau} e^{L^{2 n_{\tau}}}$

where we use the normalised expectation value

$$
\langle G\rangle_{u, v}^{\tau}=\left(\int d \mu_{u, v}^{\tau}(\omega)\right)^{-1} \int d \mu_{u, v}^{\tau}(\omega) G(\omega) .
$$

Taking derivatives, we have, for $g$ as in (70),

$$
\begin{aligned}
& \frac{d}{d u} \int d v R_{n}^{\tau}(u, v) g(v)=L^{n}\left(f^{\prime}\left(L^{n} u\right)+1\right) \int d v R_{n}^{\tau}(u, v) g(v) \\
& \quad-\int d v e^{f_{n}(u)-f_{n}(v)} \int d \mu_{u, v}^{\tau}(\omega)\left(e^{-\int_{0}^{\tau} V_{n}(\omega(s)) d s} L^{3 n} \int V^{\prime}\left(L^{n} \omega(s)\right) d s\right) g(v) \\
& \quad-L^{n} \int d v\left(f^{\prime}\left(L^{n} v\right)+1\right) R_{n}^{\tau}(u, v) g(v)+\int d v R_{n}^{\tau}(u, v) g^{\prime}(v)
\end{aligned}
$$

where the second term comes from the application of (72) (to the normalised expectation value, which obviously satisfies (71)) and the last two terms are produced by the integration by parts of $\frac{d}{d v}$. The term coming from

$$
\frac{d}{d u} e^{-\left(u-v+2 L^{n} \tau\right)^{2} / 4 \tau}
$$

is cancelled by a similar term produced by the integration by parts. 
Now we bound each of these terms for $g=\varphi, u=L u^{\prime} \leqq 0$ and $\tau=L^{2}-1$. For the first term, it is enough to use (49) and (45):

$$
L^{n+1}\left|f^{\prime}\left(L^{n+1} u^{\prime}\right)+1\right| \leqq L^{n+1} l_{n+1}\left(u^{\prime}\right) .
$$

For the last two terms, as in (70), it is enough to use (49) and:

$$
\int d v R_{n}^{L^{2}-1}\left(L u^{\prime}, v\right)\left(1+L^{n}|v|\right)^{-\frac{1}{4}} h_{n}(v) \leqq C L^{-\frac{n}{4}} h_{n+1}\left(u^{\prime}\right)
$$

for $u^{\prime} \leqq 0$. For this, we follow the proof of (49) for $u^{\prime} \leqq 0$, with the only change that, instead of (68), we use

$$
\int_{v \leqq-\varepsilon_{n}} d y e^{-c\left|x^{\prime}-\frac{y}{L}\right|}(1+|y|)^{-1-\delta}\left(1+L^{n}|v|\right)^{-\frac{1}{4}} \leqq C L^{-\frac{n}{4}}\left(1+\left|x^{\prime}\right|\right)^{-1-\delta} .
$$

This is easy to show: for $|v| \geqq 1$, use (68) and, for $|v| \leqq 1$, use the bound

$$
e^{-c\left|x^{\prime}-\frac{y}{L}\right|}(1+|y|)^{-1-\delta} \leqq C\left(1+\left|x^{\prime}\right|\right)^{-1-\delta} .
$$

Note that in (58) we have a factor $L^{-\frac{n}{3}}$ (for $\tau=L^{2}-1, t=L^{2}$ ), so (87) holds trivially, for that part of the integral, using (69). Finally, for the second term in (84), we prove (76), using parts $c$ and d of Lemma 4: in (80), we have only the first term in the RHS, because $\tau=L^{2}-1\left(t=L^{2}\right)$, and we use (69). In (81), we use, as before, part of $L^{-n p}$ to get the factor $\left(1+\left|x^{\prime}\right|\right)^{-1-\delta}$ in $h_{n+1}\left(u^{\prime}\right)$ (since $x^{\prime} \leqq 2 L^{n+1}$ for $\left.u^{\prime} \leqq 0\right)$. This completes the proof of (50) and, therefore, of (38). To prove the first estimate in (37), it is enough to show:

$$
\left|\int d v R_{n}^{\tau}(u, v) h_{n}(v)\right| \leqq L^{n \delta} h_{n, \tau+1}(u)
$$

and

$$
\left|\frac{d}{d u} \int d v R_{n}^{\tau}(u, v) \varphi(v)\right| \leqq L^{n(1+2 \delta)} l_{n}(u) h_{n, \tau+1}(u)\left(\left\|\frac{\varphi}{h_{n}}\right\|_{\infty}+\left\|\frac{\varphi^{\prime}}{\widetilde{h_{n}}}\right\|_{\infty}\right) .
$$

We follow the proof of $(49,50)$ (Lemmas 3 and 4 hold for all $\tau$ ). To prove (89), we use

$$
\int_{-\varepsilon_{n}}^{\infty} d v D_{-2 \varepsilon_{n}}^{\tau}(u, v)(1+v)^{-2-\delta} \leqq C(L)(1+u)^{-2-\delta}
$$

instead of (63), and, instead of (68),

$$
\frac{1}{\sqrt{\tau}} \int_{v \leqq-\varepsilon_{n}} d y e^{-c \frac{\left|x_{\tau}-y\right|}{\sqrt{\tau}}}(1+|y|)^{-1-\delta} \leqq C(L)\left(1+\left|x_{\tau}\right|\right)^{-1-\delta},
$$

where, see (21-23),

$$
x_{\tau}=u+2 L^{n}(\tau+1)
$$

is the variable entering $h_{n, \tau+1}$. When we use (58) (with $t=\tau+1$ ), we have to consider the second term in the RHS, for small $\tau$. However, previously, we used $L^{-\frac{n}{3}}$ only to control $C(L)$ (see the remark following Eq. (69)). So, here, we use $l_{n} \leqq 2, C(L) L^{\frac{n \delta}{4}} \leqq L^{\frac{n \delta}{2}}$ which is enough, given the factor $L^{n \delta}$ in the RHS of (89). 
To prove (90), we use

$$
\int d v R_{n}^{\tau}(u, v)\left(1+L^{n}|v|\right)^{-\frac{1}{4}} h_{n}(v) \leqq L^{n \delta} l_{n}(u) h_{n, \tau+1}(u)
$$

for all $u$, instead of $(74)$ and $(87)$, which is proven like $(74,87)$, using

$$
\int_{-\varepsilon_{n}}^{\infty} d v D_{-2 \varepsilon_{n}}^{\tau}(u, v)(1+v)^{-2-\delta}\left(1+L^{n}|v|\right)^{-\frac{1}{4}} \leqq C(L) L^{-\frac{n}{4}}(1+u)^{-2-\delta}
$$

for $u \geqq 0$, instead of (75),

$$
\frac{1}{\sqrt{\tau}} \int_{v \leqq-\varepsilon_{n}} d y e^{-\frac{\left|x_{\tau}-y\right|}{\sqrt{\tau}}}(1+|y|)^{-1-\delta}\left(1+L^{n}|v|\right)^{-\frac{1}{4}} \leqq C(L) L^{-\frac{n}{4}}\left(1+\left|x_{\tau}\right|\right)^{-1-\delta}
$$

for $u \leqq 0$, instead of (88), and $L^{-\frac{n}{4}} \leqq l_{n}(u)$. Finally, we use

$$
\int d v e^{f_{n}(u)-f_{n}(v)} k_{n}(u, v, \tau) h_{n}(v) \leqq C(L) L^{n\left(1+\frac{\delta}{2}\right)} l_{n}(u) h_{n, \tau+1}(u)
$$

for all $u$, instead of (76); (95) follows from Lemma 4 (for $t=\tau+1$ in (80)) and $(61,62)$.

Finally we shall prove (39) and the second estimate in (37), and, as before, concentrate on (39). We shall take advantage of the factor 3 in (25), as opposed to 1 in (24). This factor means that the potential barrier, for $u \leqq 0$, is higher, and, as a consequence, we shall no longer have to deal with the front in the laboratory frame (compare the first terms in (2.17) and (2.20)). To prove (39), it is enough to show (remember that $\tilde{w}$ is a multiple of $w$, see (35)):

$$
\left|\int d v Q_{n}^{L^{2}-1}\left(L u^{\prime}, v\right) w_{n}(v)\right| \leqq \frac{1}{2} L^{-(1-2 \delta)} w_{n+1}\left(u^{\prime}\right)
$$

and,

$$
\begin{aligned}
& \left|\frac{d}{d u^{\prime}} \int d v Q_{n}^{L^{2}-1}\left(L u^{\prime}, v\right) s(v)\right| \\
& \quad \leqq \frac{1}{2} L^{-(1-2 \delta)} w_{n+1}\left(u^{\prime}\right)\left(L^{(n+1)(1+\delta)}\left\|\frac{s}{w_{n}}\right\|_{\infty}+L^{1+\delta}\left\|\frac{s^{\prime}}{w_{n}}\right\|_{\infty}\right) .
\end{aligned}
$$

Instead of Lemma 3, we use

Lemma 5. For $0<\tau \leqq L^{2}-1$ and $n \geqq n_{0}$, we have

a) for $u \geqq 0$, if $v \geqq-\varepsilon_{n}$,

$$
e^{-\tau \tilde{H}_{n}}(u, v) \leqq D_{-2 \varepsilon_{n}}^{\tau}(u, v)+L^{-n p} e^{-\frac{c}{\sqrt{\tau}}(u+v)}
$$

and

$$
\int_{-\infty}^{-\varepsilon_{n}} d v Q_{n}^{\tau}(u, v)\left(1+\frac{|v|}{\sqrt{\tau}}\right) \leqq L^{-n p} e^{-\frac{c}{\sqrt{\tau}} u} e^{-L^{n} u} .
$$

b) for $u \leqq 0$, if $v \leqq-\varepsilon_{n}$,

$$
Q_{n}^{\tau}(u, v) \leqq \frac{C}{\sqrt{\tau}} \exp \left(-c \tau L^{2 n}-\frac{\left(u-v+2 L^{n} \tau\right)^{2}}{4 \tau}\right)+L^{-n p} e^{-\frac{c}{\sqrt{\tau}}(|u|+|v|)}
$$




$$
\begin{aligned}
& \text { and } \\
& \int_{-\varepsilon_{n}}^{\infty} d v Q_{n}^{\tau}(u, v) w_{n, t-\tau}(v)\left(1+\frac{|v|}{\sqrt{\tau}}\right) \leqq w_{n, t}(u)\left(L^{-\frac{n}{3}}+\chi\left(\tau \leqq L^{-\frac{n}{2}}\right)\right) e^{-\frac{c}{\sqrt{\tau}}|u|} .
\end{aligned}
$$

Moreover, the same bounds hold, with different constants, when 3 in (25) is replaced by any $\alpha>1$.

Let us prove (96). For $u^{\prime} \geqq 0$, we can use part a of Lemma 5, exactly as we used part a of Lemma 3 to prove $(49)$, the only difference being that $(61,62)$ are replaced by

$$
e^{L^{n} v} w_{n}(v) \leqq c L^{-n(1+\delta)}(1+v)^{-2-\delta}
$$

for $v \geqq-\varepsilon_{n}$, which follows immediately from (34), and

$$
e^{-L^{n+1} u^{\prime}}
$$

for $u^{\prime} \geqq 0$. The proof of (96) for $u^{\prime} \leqq 0$ is easy, given part $\mathrm{b}$ of Lemma 5: for $\tau=L^{2}-1$, even the first term of $(100)$ is small. We use $w_{n, L^{2}}\left(L u^{\prime}\right) \leqq L^{1+\delta} w_{n+1}\left(u^{\prime}\right)$ instead of (69).

To prove (97), we use the following formula, valid for $g$ as in (70) and any $u$ :

$$
\begin{aligned}
\frac{d}{d u}\left(Q_{n}^{\tau} g\right)(u)= & \frac{d}{d u} \int d v Q_{n}^{\tau}(u, v) g(v) \\
= & -\int d v e^{-L^{n_{u}}+L^{n_{v}}} \int d \mu_{u, v}^{\tau}(\omega)\left(e^{-3 \int_{0}^{\tau} V_{n}(\omega(s)) d s} L^{3 n} 3\right. \\
& \left.\times \int_{0}^{\tau} V^{\prime}\left(L^{n} \omega(s)\right) d s\right) g(v) \\
& +\int d v Q_{n}^{\tau}(u, v) g^{\prime}(v) d v .
\end{aligned}
$$

This is proven like (70), by noticing that $f(u)$ in $R$ is replaced by $-u$ here so that the first and the third terms in (70) cancel each other. To bound the second term of (104), just use (96), since $\tilde{w}$ is a multiple of $w$. For the first term, observe that, by (48), $\left|V^{\prime}\right| \leqq c V$, so that

$$
3 L^{3 n} \int_{0}^{\tau}\left|V^{\prime}\left(L^{n} \omega(s)\right)\right| d s \leqq C L^{n} e^{\int_{0}^{\tau} \frac{1}{2} V_{n}(\omega(s)) d s} .
$$

Then, we use the last statement in Lemma 5, with $\alpha=\frac{5}{2}$, and use the factor $L^{(n+1)(1+\delta)}$ in (97) to control $L^{n}$ in (105). Again, the proof of (37) is a simple modification of the proof of (39).

\subsection{Proof of Lemma 2. We need to show that}

$$
\sup _{t \in\left[1, L^{2}\right]}\left\|\int_{0}^{t-1} d \tau \int d v R_{n}^{\tau}(u, v) M(v, t-\tau)\right\|_{1, t}^{(n)} \leqq L^{-n \delta}\|(\varphi, s)\|^{(n)}
$$

and

$$
\sup _{t \in\left[1, L^{2}\right]}\left\|\int_{0}^{t-1} d \tau \int d v Q_{n}^{\tau}(u, v) N(v, t-\tau)\right\|_{2, t}^{(n)} \leqq L^{-n \delta}\|(\varphi, s)\|^{(n)}
$$


for $(\varphi, s)$ with

$$
\|(\varphi, s)\|^{(n)} \leqq 3 L^{-n(1-5 \delta)}
$$

$(M, N$ are defined in $(26,27))$.

The proof that the nonlinear terms define a contraction is similar.

To prove (106), we shall prove, for all $t \in\left[1, L^{2}\right]$,

$$
\left|\int_{0}^{t-1} d \tau \int d v R_{n}^{\tau}(u, v) M(v, t-\tau)\right| \leqq L^{-n \delta} h_{n, t}(u)\|(\varphi, s)\|^{(n)}
$$

and

$$
\left|\frac{d}{d u} \int_{0}^{t-1} d \tau \int d v R_{n}^{\tau}(u, v) M(v, t-\tau)\right| \leqq L^{-n \delta} L^{n(1+\delta)} l_{n}(u) h_{n, t}(u)\|(\varphi, s)\|^{(n)} .
$$

Let us start with the proof of (109). We use the bound $r_{n}^{-1} \leqq c\left(1+e^{L^{n} v}\right)$ to get from (34) that $w_{n} r_{n}^{-1} \leqq L^{-n}$, and, from (108),

$$
\frac{\left|s_{n}\right|}{r_{n}} \leqq L^{-n(2-\mathcal{O}(\delta))}
$$

where we use throughout the proof $\mathcal{O}(\delta)$ because we do not need to keep track of the constants multiplying $\delta$. We also have

$$
\begin{aligned}
\left|\frac{1}{1+s_{n} / r_{n}}-1\right| & \leqq L^{-n(2-\mathcal{O}(\delta))}, \\
\frac{\left|\partial s_{n}\right|}{r_{n}} & \leqq L^{-n(1-\mathcal{O}(\delta))}
\end{aligned}
$$

and

$$
\left|\partial \varphi_{n}(v, t-\tau)\right| \leqq L^{n(1+\mathcal{O}(\delta))} l_{n}(v) h_{n, t-\tau}(v)\|(\varphi, s)\|^{(n)} .
$$

We also know from (48) that $\left|q_{n}\right| \leqq c$, so, altogether,

$$
|M(v, t-\tau)| \leqq L^{n \mathcal{O}(\delta)} l_{n}(v) h_{n, t-\tau}(v)\|(\varphi, s)\|^{(n)} .
$$

Then, (109) follows by inserting (111) in (109), using the bound

$$
\int_{0}^{t-1} d \tau \int d v R_{n}^{\tau}(u, v) l_{n}(v) h_{n, t-\tau}(v) d v \leqq L^{2 n \delta} L^{-\frac{n}{4}} h_{n, t}(u)
$$

and using $L^{-\frac{n}{4}}$ to control $L^{n \mathcal{O}(\delta)}$ and to give $L^{-n \delta}$ in (109); (112) is proven by combining

$$
\int_{0}^{t-1} d \tau \int d v R_{n}^{\tau}(u, v) h_{n, t-\tau}(v) d v \leqq L^{n \delta} h_{n, t}(u),
$$

which is proven like (89), and

$$
\int_{0}^{t-1} d \tau \int d v R_{n}^{\tau}(u, v)\left(1+L^{n}|v|\right)^{-\frac{1}{4}} h_{n, t-\tau}(v) d v \leqq L^{n \delta} L^{-\frac{n}{4}} h_{n, t}(u)
$$


which is proven like (94), the only difference being that, when we use (58), we have $\int_{0}^{t-1} d \tau \chi\left(\tau \leqq L^{-\frac{n}{2}}\right) \leqq L^{-\frac{n}{2}}$ for the second term of (58), so that the result has a bound with $L^{-\frac{n}{4}}$, as in (87).

To prove (110), we would like to use $(70,84)$. However, we cannot use integration by parts as in $(70,84)$ because we have $\partial \varphi, \partial s$ in the nonlinear terms and the norms $(29,30)$ give no control over the second derivatives of $\varphi$ or $s$. We proceed as follows: write, for $u \geqq 0$,

$$
R_{n}^{\tau}(u, v)=\frac{1}{\sqrt{4 \pi \tau}} e^{f_{n}(u)-f_{n}(v)-(u-v)^{2} / 4 \tau}\left\langle e^{-\int_{0}^{\tau} V(\omega(s)) d s}\right\rangle_{u, v}^{\tau} .
$$

Taking derivatives, we have

$$
\begin{aligned}
& \left|\frac{d}{d u} \int_{0}^{t-1} d \tau \int d v R_{n}^{\tau}(u, v) M(v, t-\tau)\right| \\
& \leqq \\
& \quad L^{n O(\delta)}\|(\varphi, s)\|^{(n)}\left(\left|L^{n} f^{\prime}\left(L^{n} u\right)\right| \int_{0}^{t-1} d \tau \int d v R_{n}^{\tau}(u, v) l_{n}(v) h_{n, t-\tau}(v)\right. \\
& \quad+\int_{0}^{t-1} \int d v e^{f_{n}(u)-f_{n}(v)} k_{n}(u, v, \tau) l_{n}(v) h_{n, t-\tau}(v) \\
& \quad+\int_{0}^{t-1} d \tau \int d v \frac{|u-v|}{2 \tau} R_{n}^{\tau}(u, v) l_{n}(v) h_{n, t-\tau}(v) \\
& \quad+\int_{0}^{t-1} d \tau \int d v \frac{1}{\sqrt{4 \pi \tau}} e^{f_{n}(u)-f_{n}(v)-(u-v)^{2} / 4 \tau} \\
& \left.\quad \times\left|\frac{d}{d v}\left\langle e^{-\int_{0}^{\tau} V(\omega(s)) d s}\right\rangle_{u, v}^{\tau}\right| l_{n}(v) h_{n, t-\tau}(v)\right)
\end{aligned}
$$

where we use (72) for the normalised expectation value and then take absolute values and use (111). Now, observe that $\frac{d}{d v}\left\langle e^{-\int_{0}^{\tau} V(\omega(s)) d s}\right\rangle_{u, v}^{\tau}$ is positive by the FKG inequality $[11,14]: V$ is decreasing so $e^{-\int V}$ is increasing and the Wiener measure satisfies FKG inequalities, so $\left\langle e^{-\int_{0}^{\tau} V(\omega(s)) d s}\right\rangle_{u, v}^{\tau}$ increases if the "boundary conditions" $u$ or $v$ increases. Therefore, we may take out the absolute values in the last term of (116) and then integrate by parts, so we get a formula like (70) with $\frac{d}{d v}\left(l_{n}(v) h_{n, t-\tau}(v)\right)$ instead of $g^{\prime} ; l_{n}(v) h_{n, t-\tau}(v)$ is continuous and piecewise $C^{1}$ and

$$
\left|\frac{d}{d v}\left(l_{n}(v) h_{n, t-\tau}(v)\right)\right| \leqq L^{n} l_{n}^{2}(v) h_{n, t-\tau}(v)
$$

for $v \neq 0$ (see (31-33)). However, we get from the integration by parts another term

$$
\int_{0}^{t-1} d \tau \int d v \frac{|u-v|}{2 \tau} R_{n}^{\tau}(u, v) l_{n}(v) h_{n, t-\tau}(v)
$$

(previously, this term was cancelled by the third term in (116), but here we have taken absolute values). 
Let us bound each term of (116), by the RHS of (110), first for $u \geqq 0$ : for the first term, it is enough to use (47) (i.e. $\left.\left|f_{n}^{\prime}(u)\right| \leqq l_{n}(u)\right)$ and (112). For the second term, use $(78,79,60,61)$ and $\int_{0}^{t-1} d \tau \chi\left(\tau \leqq L^{-\frac{n}{2}}\right) \leqq L^{-\frac{n}{2}}$ for the second term of (78). Then, (110) follows for that term from

$$
L^{n \mathcal{O}(\delta)} L^{\frac{2 n}{3}} \leqq L^{n(1+\delta)} l_{n}(u) .
$$

The third term in (116) is bounded like the first one, using the exponential decay in $\frac{|u-v|}{\sqrt{\tau}}$ or in $\frac{|u|+|v|}{\sqrt{\tau}}$ in $(53,56)$ and the factor $\frac{|v|}{\sqrt{\tau}}$ in $(56)$ to control $\frac{|u-v|}{\sqrt{\tau}}$. Then we are left with the integral $\int d \tau \frac{1}{\sqrt{\tau}}$, which is finite. For the terms produced by the integration by parts, the one with $f_{n}^{\prime}(v)$ is controlled using (47), i.e. $\left|f_{n}^{\prime}(v)\right| \leqq l_{n}(v)$ and

$$
\int_{0}^{1} d \tau \int d v R_{n}^{\tau}(u, v) l_{n}^{2}(v) h_{n, t-\tau}(v) d v \leqq L^{2 n \delta} L^{-\frac{n}{12}} l_{n}(u) h_{n, t}(u),
$$

which is proven like (112), using, instead of (114),

$$
\int_{0}^{t-1} d \tau \int d v R_{n}^{\tau}(u, v)\left(1+L^{n}|v|\right)^{-\frac{1}{2}} h_{n, t-\tau}(v) d v \leqq L^{n \delta} L^{-\frac{n}{3}} h_{n, t}(u),
$$

where the worst term comes from the first term in (58). To get (119), we used $L^{-\frac{n}{3}} \leqq L^{-\frac{n}{12}} l_{n}(u)$. Still, we have $L^{-\frac{n}{12}}$ to control $L^{n \mathcal{O}(\delta)}$. The term with $\frac{|u-v|}{\tau}$ has already been discussed, while, for the term with $\frac{d}{d v}\left(l_{n}(v) h_{n, t-\tau}(v)\right)$, we use (117) and (119).

We still have to prove (110) for $u \leqq 0$. We proceed as in (116), using (82) instead of (115). All the terms are bounded as before, except

$$
\int_{0}^{t-1} d \tau \int d v \frac{\left|u-v+2 L^{n} \tau\right|}{2 \tau} R_{n}^{\tau}(u, v) l_{n}(v) h_{n, t-\tau}(v) \leqq C h_{n, t}(u)\left(L^{\frac{2 n}{3}}+L^{n\left(\frac{3}{4}+\frac{\delta}{4}\right)} l_{n}(u)\right) .
$$

Indeed, the factor $\frac{\left|u-v+2 L^{n} \tau\right|}{2 \tau}$ can be controlled as before, using the exponential decay in (57), which gives a bound like (112), while, in (58), we use the factor $L^{-n} \frac{|u-v|}{\sqrt{\tau}}$ and $\int d \tau \frac{1}{\sqrt{\tau}} \chi\left(\tau \leqq L^{-\frac{n}{2}}\right) \leqq c L^{-\frac{n}{4}}$. Then, we use (118) to get (110).

The proof of (107) follows the same pattern. We want to show:

$$
\left|\int_{0}^{t-1} d \tau \int d v Q_{n}^{\tau}(u, v) N(v, t-\tau)\right| \leqq L^{-n \delta} w_{n, t}(u)\|(\varphi, s)\|^{(n)}
$$

and

$$
\left|\frac{d}{d u} \int_{0}^{t-1} d \tau \int d v Q_{n}^{\tau}(u, v) N(v, t-\tau)\right| \leqq L^{-n \delta} L^{n(1+\delta)} w_{n, t}(u)\|(\varphi, s)\|^{(n)}
$$



$(2.5)$

Let us prove $(120,121)$ for each term in $N$, see $(27)$. For $-r_{n}\left(\partial \varphi_{n}\right)^{2}$, we use, see

$$
r_{n} \leqq c\left(1+L^{n} v\right)\left(1+e^{L^{n_{v}}}\right)^{-1}
$$

and $r_{n} h_{n} \leqq c w_{n}, h_{n} \leqq c L^{-n}$ for $v \geqq-\varepsilon_{n}$, combined with (108), to get

$$
r_{n}(v)\left(\partial \varphi_{n}\right)^{2}(v, t-\tau) \leqq L^{n \mathcal{O}(\delta)} w_{n}(v, t-\tau) l_{n}(v)\|(\varphi, s)\|^{(n)}
$$

for $v \geqq-\varepsilon_{n}$. Then, we can prove (120), for $u \geqq 0$, by using Lemma 5 , a, (102) and

$$
\int_{-\varepsilon_{n}}^{\infty} d v D_{-2 \varepsilon_{n}}^{\tau}(u, v)(1+v)^{-2-\delta}\left(1+L^{n}|v|\right)^{-\frac{1}{4}} \leqq C(L) L^{-\frac{n}{4}}(1+u)^{-2-\delta}
$$

so that we may use, as before, $L^{-\frac{n}{4}}$ to control $L^{n \mathscr{U}(\delta)}$. Now, consider (120) for $u \leqq 0$; for the integral over $v \geqq-\varepsilon_{n}$, we use (123) and (101); again, the integral over $\tau$ gives a small contribution. For $v \leqq-\varepsilon_{n}, r_{n}$ is of order one and we have, using (108),

$$
\left(\partial \varphi_{n}\right)^{2}(v, t-\tau) \leqq L^{n(1+\mathcal{O}(\delta))} h_{n, t-\tau}(v) l_{n}(v)\|(\varphi, s)\|^{(n)}
$$

Now, use (100) to prove:

$$
\int_{0}^{t-1} d \tau \int_{-\infty}^{-\varepsilon_{n}} d v Q_{n}^{\tau}(u, v) h_{n, t-\tau}(v) d v \leqq L^{-n(2-\delta)} h_{n, t}(u)
$$

and

$$
\int_{0}^{t-1} d \tau \int_{-\infty}^{-\varepsilon_{n}} d v Q_{n}^{\tau}(u, v)\left(1+L^{n}|v|\right)^{-\frac{1}{4}} h_{n, t-\tau}(v) d v \leqq L^{-n\left(2+\frac{1}{4}-\delta\right)} h_{n, t}(u) .
$$

These bounds are similar to $(113,114)$ but we use the factor $e^{-c \tau L^{2 n}}$ in $(100)$ and

$$
\int_{0}^{t-1} d \tau e^{-c \tau L^{2 n}} \leqq C L^{-2 n}
$$

to get $L^{-2 n}$ in $(125,126)$. Combining $(124-126)$, we get a bound on the integral $(120)$ over $v \leqq-\varepsilon_{n}$, for $-r_{n}\left(\partial \varphi_{n}\right)^{2}$ and $u \leqq 0$, of the form

$$
L^{-n\left(1+\frac{1}{4}-\mathcal{O}(\delta)\right)} h_{n, t}(u)\|(\varphi, s)\|^{(n)} .
$$

Then, (120) is proven for that term by observing that, for $u \leqq 0$, $L^{-n} h_{n, t}(u) \leqq C(L) L^{n \delta} w_{n, t}(u)$. The proof of (121) combines these bounds and the proof of (110) (actually, it is easier, since we do not have $l_{n}(u)$ in (121)). The term $-s_{n}\left(\partial \varphi_{n}\right)^{2}$ in (27) can be treated in the same way, since $s_{n}$ is smaller than $r_{n}$.

The final term to consider is $-3 r_{n} L^{2 n} s_{n}^{2}$, since the term $-L^{2 n} s_{n}^{3}$ is smaller. Following the same strategy as before, we use, instead of $(123,124)$, the bound:

$$
r_{n}(v) L^{2 n} s_{n}^{2}(v, t-\tau) \leqq L^{n \mathcal{O}(\delta)}\left(1+L^{n} v\right)^{2}\left(1+e^{L^{n} v}\right)^{-2} w_{n, t-\tau}(v)\|(\varphi, s)\|^{(n)},
$$

which follows from $(108,122)$ and $w_{n} \leqq L^{-n}$. Because of the factor $\left(1+L^{n} v\right)^{2}\left(1+e^{L^{n} v}\right)^{-2}$, we get an extra $L^{-n}$ when we integrate over $v \geqq 0$, which is used to control $L^{n \mathcal{O}(\delta)}$, and we get $L^{-2 n}$ from (127) when we integrate over $v \leqq-\varepsilon_{n}$; the integrals over $-\varepsilon_{n} \leqq v \leqq 0$ are controlled using (98), (101), and $\int_{0}^{t-1} d \tau \int_{-\varepsilon_{n}}^{0} d v D_{-2 \varepsilon_{n}}^{\tau}(u, v) \leqq c \varepsilon_{n}$. 


\section{Appendix}

Proof of Lemma 3. To prove (3.53), we use the path space representation (3.51). For each path $\omega$, let $t_{1}$ be the time of the first visit to $-2 \varepsilon_{n}$, if $\omega$ visits $-2 \varepsilon_{n}$ before time $\tau$ and let $t_{2}$ be the time of the first visit to $-\frac{3}{2} \varepsilon_{n}$ after $t_{1}$ (since $v \geqq-\varepsilon_{n}, t_{2} \leqq \tau$ if $\left.t_{1}<\tau\right)$. So, by conditioning,

$$
\begin{aligned}
e^{-\tau H_{n}}(u, v)= & \int_{0}^{\tau} d t_{1} \int_{t_{1}}^{\tau} d t_{2} \int d \mu_{u,-2 \varepsilon_{n}}^{t_{1}}\left(\omega>-2 \varepsilon_{n}\right) d \mu_{-2 \varepsilon_{n},-\frac{3}{2} \varepsilon_{n}}^{t_{2}-t_{1}}\left(\omega<-\frac{3}{2} \varepsilon_{n}\right) \\
& \cdot d \mu_{-\frac{3}{2} \varepsilon_{n}, v}^{\tau-t_{2}}(\omega) e^{-\int_{0}^{\tau} V_{n}(\omega(s)) d s} \\
& +\int d \mu_{u, v}^{\tau}(\omega) \chi\left(\omega>-2 \varepsilon_{n}\right) e^{-\int_{0}^{\tau} V_{n}(\omega(s)) d s},
\end{aligned}
$$

where $d \mu_{a, b}^{t}(\omega>b)$ denotes the measure on paths with $\omega(0)=a, \omega(t)=b$ and $\omega(s)>b$ for $s<t$, defined by:

$$
\int F(\omega) d \mu_{a, b}^{t}(\omega>b)=\left.2 \frac{d}{d x} \int F(\omega) d \mu_{a, x}^{t}(\omega)\right|_{x=b} .
$$

For our $F$, the RHS is $C^{\infty}$ in $x$ and this indeed defines an expectation. This equation may be derived by the method of images from random walks. We will have below positive $F$ 's estimated from above by constants, and the only thing we need to know is the formula for $F=1$,

$$
\int d \mu_{a, b}^{t}(\omega>b)=\frac{(a-b)}{\left(4 \pi t^{3}\right)^{1 / 2}} e^{-\frac{(a-b)^{2}}{4 t}}
$$

which is the probability density that $t$ is the first time at which $\omega$, starting from $a$, reaches $b$.

Using $V_{n} \geqq 0$, the second term in (1) is bounded by $D_{-2 \varepsilon_{n}}^{\tau}(u, v)$ and the formula (3.54) follows from the method of images. In the first term, we use

$$
\int_{0}^{\tau} V_{n}(\omega(s)) d s \geqq L^{2 n}\left(t_{2}-t_{1}\right)-C
$$

which holds because, for $s \in\left[t_{1}, t_{2}\right], \omega(s)<-\frac{3}{2} \varepsilon_{n}, V(x) \geqq 1-C e^{-c|x|}$, and $L^{2 n} e^{-c \varepsilon_{n} L^{n}} \leqq C / L^{2} \leqq C / \tau$ for $k$ large in (3.52). So, using (2), (3), the first term in (1) can be bounded by

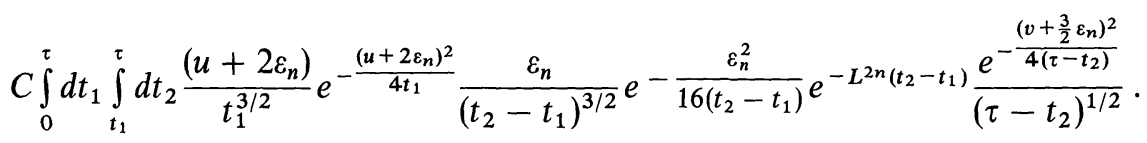

Now we use the following three bounds. First,

$$
\frac{e^{-\frac{\left(v+\frac{3}{2} \varepsilon_{n}\right)^{2}}{4\left(\tau-t_{2}\right)}}}{\left(\tau-t_{2}\right)^{1 / 2}} \leqq \frac{C e^{-c \frac{|v|}{\sqrt{\tau}}}}{\varepsilon_{n}}
$$

which holds because $v \geqq-\varepsilon_{n}, \tau-t_{2} \leqq \tau$. Secondly,

$$
\int_{0}^{\infty} d s \frac{|z|}{s^{3 / 2}} e^{-\frac{z^{2}}{4 s}} e^{-\alpha s} \leqq C e^{-\sqrt{\alpha}|z|}
$$


which follows by shifting $s$ by the location of the maximum of the exponent $\frac{|z|}{2 \sqrt{\alpha}}$. We use (6) with $z=\varepsilon_{n}$, and $s=4\left(t_{2}-t_{1}\right), \alpha=\frac{L^{2 n}}{4}$. Thirdly,

$$
\int_{0}^{\tau} d s \frac{|z|}{s^{3 / 2}} e^{-\frac{z^{2}}{4 s}} \leqq C e^{-c \frac{|z|}{\sqrt{\tau}}}
$$

which follows also from a change of variable, $s^{\prime}=\frac{s}{z^{2}}$. We use (7) with $z=u+2 \varepsilon_{n}$ and $s=t_{1}$.

Using $(5,6,7)$, we get

$$
\text { (4) } \leqq \frac{C}{\varepsilon_{n}} e^{-c L^{n_{\varepsilon_{n}}}} e^{-\frac{c}{\sqrt{\tau}}(u+v)} \leqq L^{-n p} e^{-\frac{c}{\sqrt{\tau}}(u+v)}
$$

for $k$ large in (3.52). This completes the proof of (3.53).

To prove (3.56), we use (3.46):

$$
R_{n}^{\tau}(u, v) \leqq C e^{-f_{n}(v)}\left(e^{-\tau H_{n}}\right)(u, v)\left(1+L^{n} u\right)^{-1}
$$

and, by $(3.44,3.52)$,

$$
-f_{n}(v) \leqq L^{n} v+c \leqq-\varepsilon_{n} L^{n}+c=-k n \log L+c
$$

for $v \leqq-\varepsilon_{n}$. Then we use $V_{n} \geqq 0$, to bound

$$
e^{-\tau H_{n}}(u, v) \leqq \int d \mu_{u, v}^{\tau}(\omega)=\frac{e^{-\frac{(u-v)^{2}}{4 \tau}}}{\sqrt{4 \pi \tau}} .
$$

And, since $u \geqq 0, v \leqq-\varepsilon_{n}$, we get $(3.56)$ from $(9,10,11)$.

To prove (3.57) we use again (3.51). Let $t_{1}$ be the time of the first visit of $\omega$ to $-\frac{\varepsilon_{n}}{2}$, if $\omega$ visits $-\frac{\varepsilon_{n}}{2}$ at all. We have

$$
\begin{aligned}
R_{n}^{\tau}(u, v)= & \exp \left(f_{n}(u)-f_{n}(v)\right)\left(\int d \mu_{u, v}^{\tau}(\omega) e^{-\int_{0}^{\tau} V_{n}(\omega(s)) d s} \chi\left(\omega<-\frac{\varepsilon_{n}}{2}\right)\right. \\
& \left.+\int_{0}^{\tau} d t_{1} \int d \mu_{u,-\frac{\varepsilon_{n}}{2}}^{t_{1}}\left(\omega<-\frac{\varepsilon_{n}}{2}\right) d \mu_{-\frac{\varepsilon_{n}}{2}, v}^{\tau-t_{1}}(\omega) e^{-\int_{0}^{\tau} V_{n}(\omega(s)) d s}\right) .
\end{aligned}
$$

We use (3.44):

$$
f_{n}(u)-f_{n}(v) \leqq-L^{n}(u-v)+c=L^{n}(|u|-|v|)+c
$$

for $u, v$ less than zero; for the first term in (12), we have, as in (3),

$$
\int_{0}^{\tau} V_{n}(\omega(s)) d s \geqq \tau L^{2 n}-C
$$

because $\omega(s)<-\frac{\varepsilon_{n}}{2}$. Using the equality in (11), the first term of (12) is bounded by the first one in (3.57). 
For the second term in (12), we have

$$
\int_{0}^{\tau} V_{n}(\omega(s)) d s \geqq t_{1} L^{2 n}-C
$$

and

$$
\int d \mu_{-\frac{\varepsilon_{n}}{2}, v}^{\tau-t_{1}}(\omega) \leqq C\left(\tau-t_{1}\right)^{-\frac{1}{2}} e^{-\frac{c|v|}{\sqrt{\tau-t_{1}}} \leqq} \frac{C}{\varepsilon_{n}} e^{-\frac{c}{\sqrt{\tau}}|v|},
$$

which holds because $v \leqq-\varepsilon_{n}$. Using $(2)$ and $(15,16)$ we bound the second integral in (12) by

$$
\frac{C}{\varepsilon_{n}} e^{-\frac{c}{\sqrt{\tau}}|v|} \int_{0}^{\tau} d t_{1} \frac{\left|u+\frac{\varepsilon_{n}}{2}\right|}{t_{1}^{3 / 2}} e^{-\frac{\left(v+\frac{\varepsilon_{n}}{2}\right)^{2}}{4 t_{1}}-t_{1} L^{2 n}}
$$

which, using (6) with $z=u+\frac{\varepsilon_{n}}{2}, \alpha=L^{2 n}$ is less than

$$
\frac{C}{\varepsilon_{n}} e^{-\frac{c}{\sqrt{\tau}}|v|} e^{-L^{n}|u|+L^{n \frac{\varepsilon_{n}}{2}}}
$$

since $u \leqq 0$. Combining this with (13), and remembering that $v \leqq-\varepsilon_{n}$, i.e. $L^{n} v+L^{n} \frac{\varepsilon_{n}}{2} \leqq-L^{n} \frac{\varepsilon_{n}}{2}$, the second term of (12) is bounded by

$$
L^{-n p} e^{-\frac{c}{\sqrt{\tau}}|v|}
$$

which is the second term of (3.57) for $x>0$. For $x<0$, we have, see (3.21-3.23) $|u|=2(\tau+1) L^{n}+|x|$ and the factor $\frac{\left(u+\varepsilon_{n} / 2\right)^{2}}{4 t_{1}}+t_{1} L^{2 n}$ in (17) reaches its minimum at $t_{1}=\tau$, where

$$
\frac{\left(u+\frac{\varepsilon_{n}}{2}\right)^{2}}{4 \tau}+\tau L^{2 n} \geqq L^{n}|u|-\frac{L^{n} \varepsilon_{n}}{2}+c \frac{|x|}{\sqrt{\tau}}
$$

which then gives (3.57).

Finally, we prove (3.58). Let $t_{1}$ be the time of the first visit of $\omega$ to zero. The LHS of (3.58) is bounded by

$$
\begin{aligned}
& C L^{-n(1+\delta)} e^{L^{n}|u|}\left(\int_{-\varepsilon_{n}}^{\infty} d v\left(1+L^{-n} \frac{|u-v|}{\sqrt{\tau}}\right) \int_{0}^{\tau} d t_{1}\right. \\
& \quad \times \int d \mu_{u, 0}^{t_{1}}(\omega<0) e^{-\int_{0}^{\tau} V_{n}(\omega(s)) d s} d \mu_{0, v}^{\tau-t_{1}}(\omega) \\
& \left.\quad+\int_{-\varepsilon_{n}}^{0} d v e^{-L^{n}|v|}\left(1+L^{-n} \frac{|u-v|}{\sqrt{\tau}}\right) \int d \mu_{u, v}^{\tau}(\omega) \chi(\omega<0) e^{-\int_{0}^{\tau} V_{n}(\omega(s)) d s}\right),
\end{aligned}
$$

where the second term collects walks that do not visit zero, in which case $v$ has to be negative. We used:

$$
e^{-f_{n}(v)} h_{n}(v) \leqq C L^{-n(1+\delta)}
$$


for $v \geqq-\varepsilon_{n}$ (see (3.61)), in the first term and

$$
e^{-f_{n}(v)} h_{n}(v) \leqq C L^{-n(1+\delta)} e^{-L^{n}|v|}
$$

for $-\varepsilon_{n} \leqq v \leqq 0$ in the second term.

We shall bound each term of (21) by

$$
C(L) L^{-n(1+\delta)}\left(L^{-n\left(\frac{1}{3}+\delta\right)}+L^{\frac{n \delta}{8}} l_{n}(u) \chi\left(\tau \leqq L^{-n / 2}\right)\right) e^{-\frac{\mathcal{c}}{\sqrt{\tau}}|x| \chi(x<0)}
$$

and, to get (3.58), use

$$
L^{-n(1+\delta)} e^{-\frac{c}{2 \sqrt{\tau}}|x| \chi(x<0)} \leqq C(L) h_{n, t}(u)
$$

for $u<0$, which holds since $x \leqq 2 t L^{n}$ for $x>0, u<0$, and then, use (3.43).

For the first term of (21), we write

$$
\int_{0}^{\tau} V_{n}=\int_{0}^{t_{1}} V_{n}+\int_{t_{1}}^{\tau} V_{n}
$$

and we have, by (3.53),

$$
\int d \mu_{0, v}^{\tau-t_{1}}(\omega) e^{-\int_{t_{1}}^{\tau} V_{n}} \leqq D_{-2 \varepsilon_{n}}^{\tau-t_{1}}(0, v)+L^{-n p} e^{-c \frac{|v|}{\sqrt{\tau}}}
$$

Besides, from the explicit formula (3.54), one gets

$$
\int_{-\varepsilon_{n}}^{\infty} d v D_{-2 \varepsilon_{n}}^{\tau-t_{1}}(0, v)\left(1+\frac{|v|}{\sqrt{\tau}}\right) \leqq C \min \left(\frac{\varepsilon_{n}}{\sqrt{\tau-t_{1}}}, 1\right)
$$

and, after integration over $v$, the second term in (24) is smaller. Also, we shall prove below that

$$
\int d \mu_{u, 0}^{t_{1}}(\omega<0) e^{-\int_{0}^{t_{1}} V_{n}} \leqq \begin{cases}C \frac{|u|}{t_{1}^{3 / 2}} e^{-\frac{u^{2}}{4 t_{1}}-L^{n}|u|-c t_{1} L^{2 n}} & \text { if } t_{1}>\frac{4|u|}{L^{n}} \\ C \frac{|u|}{t_{1}^{3 / 2}} e^{-\frac{u^{2}}{4 t_{1}}-t_{1} L^{2 n}} & \text { if } t_{1} \leqq \frac{4|u|}{L^{n}}\end{cases}
$$

Let us consider $\tau \leqq \frac{4|u|}{L^{n}}$. Using (24-26), we bound the first integral in (21) by

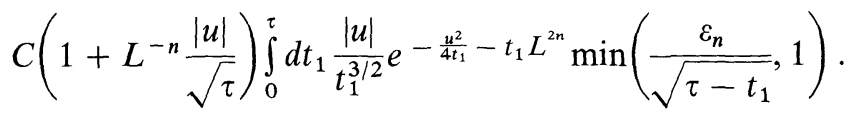

Consider first $|u| \leqq L^{-n}$. Then we bound the minimum in (27) by 1 , the integral is bounded by $e^{-c \frac{|u|}{\sqrt{\tau}}}$, using (7), and this is all we need in (22), since here $x>0$, $\tau \leqq 4 L^{-2 n} \leqq L^{-\frac{n}{2}}$ and $l_{n}(u) \geqq c$ for $|u| \leqq L^{-n}$. So, let $|u| \geqq L^{-n}$, and bound the minimum in (27) by $\frac{\varepsilon_{n}}{\sqrt{\tau-t_{1}}}$. Then, change variables:

$$
u=L^{-n} \tilde{u}, \quad t_{1}=\frac{|u| s}{2 L^{n}}=\frac{|\tilde{u}| s}{2 L^{2 n}}, \quad \tau=\frac{|u| \sigma}{2 L^{n}}=\frac{|\tilde{u}| \sigma}{2 L^{2 n}} .
$$


Note, that now $|\tilde{u}| \geqq 1$. We get the integral

$$
\frac{\varepsilon_{n} L^{n}}{\sqrt{|\tilde{u}|}}\left(1+c L^{-n} \frac{\sqrt{|\tilde{u}|}}{\sqrt{\sigma}}\right) \int_{0}^{\sigma} d s \frac{\sqrt{|\tilde{u}|} e^{-\frac{|\tilde{u}|}{2}\left(s+\frac{1}{s}\right)}}{s^{3 / 2} \sqrt{\sigma-s}}
$$

which we want to bound, see $(21,22)$, for $x \geqq 0$, by

$$
e^{-|\tilde{u}|} L^{-n\left(\frac{1}{3}+\delta\right)} \text { for } \sigma \geqq \frac{2 L^{\frac{3 n}{2}}}{|\tilde{u}|}
$$

and by

$$
\frac{L^{\frac{n \delta}{s}} e^{-|\tilde{u}|}}{(1+|\tilde{u}|)^{1 / 4}} \text { for } \sigma \leqq \frac{2 L^{\frac{3 n}{2}}}{|\tilde{u}|} .
$$

We divide the integral in (28) into $|s-1| \leqq \frac{1}{4}$ and $|s-1| \geqq \frac{1}{4}$. For the first part, a Gaussian integral around $s=1$ gives

$$
\int_{3 / 4}^{5 / 4} d s \frac{\sqrt{|\tilde{u}|} e^{-\frac{|\tilde{u}|}{2}\left(s+\frac{1}{s}\right)}}{\sqrt{\sigma-s}} \leqq c|\tilde{u}|^{1 / 4} e^{-|\tilde{u}|}
$$

and, if $\sigma \geqq \frac{3}{4}, \tau \leqq L^{2}$ implies that $|\tilde{u}| \leqq C(L) L^{2 n}$, so that $\left(1+c L^{-n} \frac{\sqrt{|\tilde{u}|}}{\sqrt{\sigma}}\right) \leqq C(L)$, while

$$
\int_{0}^{\sigma} d s \frac{\sqrt{|\tilde{u}|} e^{-\frac{|\tilde{u}|}{2}\left(s+\frac{1}{s}\right)}}{s^{3 / 2} \sqrt{\sigma-s}} \chi\left(|s-1| \geqq \frac{1}{4}\right) \leqq c e^{-|\tilde{u}|},
$$

where we use $s+\frac{1}{s} \geqq 2+\frac{c}{s}$ for $|s-1| \geqq \frac{1}{4}$ and we use the factor $\exp \left(-\frac{c|\tilde{u}|}{2 s}\right)$ to control $\frac{\sqrt{|\tilde{u}|}}{s^{3 / 2}}$ or $\frac{\sqrt{|\tilde{u}|}}{\sqrt{\sigma}}$ (since $\left.|\tilde{u}| \geqq 1\right)$.

Using $(31,32)$, we get that $(28)$ is bounded by

$$
C(L) \varepsilon_{n} L^{n}|\tilde{u}|^{-\frac{1}{4}} e^{-|\tilde{u}|}
$$

which is always less than (30) since $|\tilde{u}| \geqq 1$. And, for $\sigma \geqq \frac{2 L^{\frac{3 n}{2}}}{|\tilde{u}|}$, we have $|\tilde{u}| \geqq c L^{\frac{3 n}{2}}$, since $\tau \leqq \frac{4|u|}{L^{n}}$ means $\sigma \leqq 8$. So, $|\tilde{u}|^{-\frac{1}{4}} \leqq L^{-\frac{3 n}{8}}$ and, inserting this in (33), gives (29), for $\delta$ small.

For $x \leqq 0$, we have $|u| \geqq 2(\tau+1) L^{n}, \tau \leqq L^{2}-1$, so that $\sigma \leqq 1-\frac{c}{L^{2}}$. Since $\sigma$ is bounded away from one, we can improve the bound in $(31,32)$ into $c e^{-|\tilde{u}|\left(1+\frac{c}{L^{2}}\right)}$. But $\frac{|\tilde{u}|}{L^{2}} \geqq|x|$ for $x<0$, so we get the exponential decay needed in (22). 
For $\tau \geqq \frac{4|u|}{L^{n}}$, we get from (24-26) and from $L^{-n} \frac{\sqrt{|u|}}{\sqrt{\tau}} \leqq \sqrt{\tau} \leqq L$ an upper bound on the first integral in (21) of the form:

$$
\begin{aligned}
C(L) & \left(\int_{0}^{4 L-n|u|} d t_{1} \frac{|u|}{t_{1}^{3 / 2}} e^{-\frac{u^{2}}{4 t_{1}}-t_{1} L^{2 n}} \min \left(\frac{\varepsilon_{n}}{\sqrt{\tau-t_{1}}}, 1\right)\right. \\
& \left.+e^{-L^{n}|u|} \int_{4 L^{-n|u|}}^{\tau} d t_{1} \frac{|u|}{t_{1}^{3 / 2}} e^{-\frac{u^{2}}{4 t_{1}}-c t_{1} L^{2 n}} \min \left(\frac{\varepsilon_{n}}{\sqrt{\tau-t_{1}}}, 1\right)\right) .
\end{aligned}
$$

The first term can be analyzed as we did with (27) and the second term is always bounded by $e^{-L^{n}|u|} e^{-c L^{n}|u|}$ and is bounded by $c \varepsilon_{n} L^{\frac{n}{4}} e^{-L^{n}|u|} e^{-c L^{n}|u|}$ for $\tau \geqq L^{\frac{-n}{2}}$. These bounds are less than $(29,30)$.

For the second integral in (21), we introduce $1=\chi(v>u)+\chi(v \leqq u)$ and, for $v>u$, we let $t_{1}$ be the time of the first visit to $v$. Then, we can bound that integral by

$$
\begin{aligned}
& \int_{-\varepsilon_{n}}^{0} d v \chi(v>u) e^{-L^{n}|v|}\left(1+L^{-n} \frac{|u-v|}{\sqrt{\tau}}\right) \int_{0}^{\tau} d t_{1} d \mu_{u, v}^{t_{1}}(\omega<v) e^{-\int_{0}^{\tau} V_{n}(\omega(s)) d s} d \mu_{v, v}^{\tau-t_{1}}(\omega) \\
& \quad+c \int_{-\varepsilon_{n}}^{0} d v \chi(v \leqq u) e^{-L^{n}|v|}\left(1+L^{-n} \frac{|u-v|}{\sqrt{\tau}}\right) \frac{e^{-\frac{(u-v)^{2}}{4 \tau}}}{\sqrt{\tau}}
\end{aligned}
$$

The second term is bounded by

$$
c \min \left(1, \frac{L^{-n}}{\sqrt{\tau}}\right) e^{-L^{n}|u|}
$$

which gives a contribution to (22) since, for $|u| \leqq \varepsilon_{n}, l_{n}(u) \geqq\left(1+L^{n}|u|\right)^{-\frac{1}{4}} \geqq L^{-\frac{n \delta}{8}}$. For the first term in (34), we use:

$$
\begin{aligned}
& \int d \mu_{u, v}^{t_{1}}(\omega<v) e^{-\int_{0}^{\tau} V_{n}(\omega(s)) d s} \\
& \leqq \begin{cases}C \frac{|u-v|}{t_{1}^{3 / 2}} \exp \left(-\frac{(u-v)^{2}}{4 t_{1}}-L^{n}(|u-v|)-c t_{1} L^{2 n}\right) & \text { if } t_{1}>\frac{4|u-v|}{L^{n}} \\
C \frac{|u-v|}{t_{1}^{3 / 2}} \exp \left(-\frac{(u-v)^{2}}{4 t_{1}}-t_{1} L^{2 n}\right) & \text { if } t_{1} \leqq \frac{4|u-v|}{L^{n}}\end{cases}
\end{aligned}
$$

and argue as for the first integral in (21).

So, we are left with the proof of (26). The proof of (35) is similar. Consider first $t_{1} \geqq \frac{4|u|}{L^{n}}$. Using (3.44) we have $r_{0}^{2}(u) \geqq 1-c \lambda \geqq 1 / 2$ for $u<0$, so

$$
\int_{0}^{t_{1}} V_{n}(\omega(s)) d s \geqq \frac{L^{2 n} t_{1}}{2} \geqq L^{n}|u|+c t_{1} L^{2 n}
$$

which, combined with (2), gives (26) for that case. 
For $t_{1} \leqq \frac{4|u|}{L^{n}}$, we write

$$
\int_{0}^{t_{1}} V_{n}(\omega(s)) d s \geqq L^{2 n} t_{1}-\lambda \int W_{n}(\omega(s)) d s,
$$

where

$$
\left|W_{n}(\omega(s))\right| \leqq L^{2 n} e^{-c L^{n}|\omega(s)|}
$$

and, as in (3.44), the origin of the coordinates is chosen so that $\lambda$ is small enough. So to prove (26), we have to show (see (2))

$$
\left\langle e^{\lambda \int_{0}^{t_{1}} W_{n}(\omega(t)) d s}\right\rangle \leqq C,
$$

where the expectation value and the probability $P$ below refer to the measure $d \mu_{u, 0}^{t_{1}}(\omega<0)$. We bound the LHS of (39) by

$$
\sum_{m \geqq 0} e^{\lambda(m+1)} P\left(\left|\int_{0}^{t_{1}} W_{n}(\omega(s)) d s\right| \in[m, m+1]\right) .
$$

Define $t_{k}(\omega)=\left\{\left\{s \mid \omega(s) \in\left[\frac{-k}{L^{n}}, \frac{-k+1}{L^{n}}\right]\right\} \mid\right.$ for $k \geqq 1$. By (38), $\int_{0}^{t_{1}} W_{n}(\omega(s)) d s$ $\leqq L^{2 n} \sum_{k} t_{k} e^{-c(k-1)}$; so, we can bound (40) by

$$
\sum_{m \geqq 0} e^{\lambda(m+1)} \sum_{k \geqq 1} P\left(t_{k}(\omega) \geqq \alpha m k L^{-2 n}\right),
$$

where $\alpha$ is such that $\alpha \sum_{k} k e^{-c(k-1)} \leqq 1$. We shall prove that

$$
P\left(t_{k}(\omega) \geqq \alpha m k L^{-2 n}\right) \leqq C e^{-c \alpha m k}
$$

for $m$ large. This then proves (39) for $\lambda$ small enough.

Write

$$
\int d \mu_{u, 0}^{t_{1}}(\omega<0)=\int_{0}^{t_{1}} d s \int d \mu_{u, \frac{-k}{L^{n}}}^{t_{1}-s}\left(\omega<-\frac{k}{L^{n}}\right) d \mu_{-\frac{k}{L^{n}}, 0}^{s}(\omega<0)
$$

i.e. $t_{1}-s$ is the time of the first visit of $\omega$ to $\frac{-k}{L^{n}}$. Clearly,

$$
\chi\left(t_{k}(\omega) \geqq \alpha m k L^{-2 n}\right) \leqq \chi\left(s(\omega) \geqq \alpha m k L^{-2 n}\right) .
$$

So that to prove (41) we will show (see (2))

$$
\int_{\alpha m k L^{-2 n}}^{t_{1}} d s \int d \mu_{u, \frac{-k}{L^{n}}}^{t_{1}-s}\left(\omega<-\frac{k}{L^{n}}\right) d \mu_{-\frac{k}{L^{n}}, 0}^{s}(\omega<0) \leqq C e^{-c \alpha m k} \frac{|u|}{t_{1}^{3 / 2}} e^{-\frac{u^{2}}{4 t_{1}}},
$$

where the LHS is

$$
C \int_{\alpha m k L^{-2 n}}^{t_{1}} d s \frac{\left|u+\frac{k}{L^{n}}\right|}{\left(t_{1}-s\right)^{3 / 2}} e^{-\frac{\left(u+\frac{k}{L^{n}}\right)^{2}}{4\left(t_{1}-s\right)}} \frac{k}{L^{n} s^{3 / 2}} e^{-\left(\frac{k}{L^{n}}\right)^{2} / 4 s} .
$$


Consider (43) for $u \leqq-\frac{2 k}{L^{n}}$, otherwise $t_{1} \leqq \frac{4|u|}{L^{n}} \leqq \frac{8 k}{L^{2 n}} \leqq \frac{\alpha m k}{L^{2 n}}$ for $m$ large, and (43) is trivial. Now use $t_{1}-s=t_{1}\left(1-\frac{s}{t_{1}}\right), \frac{1}{1-s / t_{1}} \geqq 1+\frac{s}{t_{1}}, 1+\frac{s}{t_{1}} \leqq 2$, to get

$$
\frac{\left(u+\frac{k}{L^{n}}\right)^{2}}{4 t_{1}\left(1-\frac{s}{t_{1}}\right)} \geqq \frac{u^{2}}{4 t_{1}}+\frac{s}{4 t_{1}^{2}} u^{2}-\frac{|u| k}{t_{1} L^{n}}
$$

which, for $t_{1} \leqq \frac{4|u|}{L^{n}}$ and $s \geqq \alpha m k L^{-2 n}$, i.e. for $\frac{s|u|}{t_{1}} \geqq \frac{\alpha m k L^{-n}}{4}$, is, for $m$ large, bigger than

$$
\frac{u^{2}}{4 t_{1}}+\frac{s}{8 t_{1}^{2}} u^{2} \geqq \frac{u^{2}}{4 t_{1}}+c \alpha m k
$$

Now consider separately the integral in (44) over $s$ so that $t_{1}-s \geqq \eta t_{1}$ and $t_{1}-s \leqq \eta t_{1}$, where $\eta$ is small, say less than $\frac{1}{16}$. For $t_{1}-s \geqq \eta t_{1}$ we use $\left|u+\frac{k}{L^{n}}\right|$ $\leqq|u|\left(\right.$ since $\left.u \leqq-\frac{2 k}{L^{n}}\right)$ and (46) to bound that integral by

$$
C \frac{|u|}{t_{1}^{3 / 2}} e^{-\frac{u^{2}}{4 t_{1}}} e^{-c \alpha m k} \int d s \frac{k}{L^{n} s^{3 / 2}} e^{-\left(\frac{k}{L^{n}}\right)^{2} / 4 s}
$$

where the last integral is of order one by (7). So we get a contribution to (43). For $t_{1}-s \leqq \eta t_{1}$, with $\eta$ small, we have for $u \leqq-\frac{2 k}{L^{n}},\left|u+\frac{k}{L^{n}}\right| \geqq \frac{1}{2}|u|$, and

$$
\frac{\left(u+\frac{k}{L^{n}}\right)^{2}}{4\left(t_{1}-s\right)} \geqq \frac{\left(u+\frac{k}{L^{n}}\right)^{2}}{8\left(t_{1}-s\right)}+\frac{u^{2}}{2 t_{1}}
$$

since $s \leqq t_{1}, \frac{u^{2}}{4 t_{1}} \geqq \frac{s u^{2}}{4 t_{1}^{\frac{2}{1}}} \geqq c \alpha m k$, where the second inequality comes from $t_{1} \leqq \frac{4|u|}{L^{n}}$, $s \geqq \alpha m k L^{-2 n}$. So we may bound the integral (44) over $t_{1}-s \leqq \eta t_{1}$, by

$$
C e^{-\frac{u^{2}}{4 t_{1}}} e^{-c \alpha m k} \int_{0}^{t_{1}} d s \frac{\left|u+\frac{k}{L^{n}}\right|}{\left(t_{1}-s\right)^{3 / 2}} e^{-\frac{\left(u+\frac{k}{L^{n}}\right)^{2}}{8\left(t_{1}-s\right)}} \frac{k}{L^{n} s^{3 / 2}} e^{-\left(\frac{k}{L^{n}}\right)^{2} / 8 s}
$$

but, going back to (42) and using (2), we see that the last integral is proportional to

$$
\frac{|u|}{t_{1}^{3 / 2}} e^{-\frac{u^{2}}{8 t_{1}}} \leqq \frac{|u|}{t_{1}^{3 / 2}}
$$

(we apply (42) to a Wiener measure with a diffusion constant whose value is twice the original one); (48) inserted in (47) gives (43). 
Proof of Lemma 4. To prove (3.78) consider first $u \geqq 2 \varepsilon_{n}$ and let $t_{1}$ be the time of the first visit to $\varepsilon_{n}$. We write

$$
\begin{aligned}
k_{n}(u, v, \tau) \leqq & c L^{n} \int_{0}^{\tau} d t_{1} \int d \mu_{u, \varepsilon_{n}}^{t_{1}}\left(\omega>\varepsilon_{n}\right) d \mu_{\varepsilon_{n, v}}^{\tau-t_{1}}(\omega) e^{-\frac{1}{2} \int_{t_{1}}^{\tau} V_{n}(\omega(s)) d s} \\
& +L^{3 n} \int d \mu_{u, v}^{\tau}(\omega) \chi\left(\omega>\varepsilon_{n}\right) \int_{0}^{\tau}\left|V^{\prime}\left(L^{n} \omega(s)\right)\right| d s
\end{aligned}
$$

where we use (3.105) and $V \geqq 0$.

We can see that $(24,25)$ hold with 0 replaced by $\varepsilon_{n}$ and $V$ by $\frac{1}{2} V$ so that the first term of (49) gives a bound on $\int_{-\varepsilon_{n}}^{\infty} d v k_{n}(u, v, \tau)$ of the form

$$
C L^{n} \int_{0}^{\tau} d t_{1} \frac{\left|u-\varepsilon_{n}\right|}{t_{1}^{3 / 2}} e^{-\frac{\left(u-\varepsilon_{n}\right)^{2}}{4 t_{1}}} \min \left(\frac{\varepsilon_{n}}{\sqrt{\tau-t_{1}}}, 1\right)
$$

where we used also (2). This integral is itself bounded by

$$
\frac{L^{\frac{n \delta}{4}}}{\sqrt{\tau}} e^{-\frac{c\left|u-\varepsilon_{n}\right|}{\sqrt{\tau}}}
$$

which gives a contribution to the RHS of (3.78): for $\tau \leqq L^{\frac{-n}{2}}$, we use $\frac{1}{\sqrt{\tau}} \leqq \frac{C}{\left|u-\varepsilon_{n}\right|} e^{\frac{c}{2} \frac{\left|u-\varepsilon_{n}\right|}{\sqrt{\tau}}}$ and $\left|u-\varepsilon_{n}\right|^{-1} \leqq L^{n} l_{n}(u)$ for $u \geqq 2 \varepsilon_{n}$.

For the second term in (49), we have

$$
\int_{0}^{\tau} \mid V^{\prime}\left(L^{n} \omega(s) \mid d s \leqq L^{-n p}\right.
$$

since $\omega \geqq \varepsilon_{n}$. To obtain the factor $e^{-\frac{c}{L} u}$ in (3.78), let $x=x(\omega)$ be the point closest to zero which is visited by $\omega$. Then we have

$$
\int_{0}^{\tau}\left|V^{\prime}\left(L^{n} \omega(s)\right)\right| d s \leqq L^{-n p} e^{-c L^{n} x},
$$

and we get a decay $e^{-\frac{c}{\sqrt{\tau}}|u-x|}$ from the Wiener measure, for the paths going in time less than $\tau$ from $u$ to $x$. Finally, for $u \leqq 2 \varepsilon_{n}$, we use $(3.105)$ and $(24,25)$ with 0 replaced by $u$ and $V$ replaced by $\frac{1}{2} V$, to get

$$
\int d v k_{n}(u, v, \tau) \leqq c L^{n} \min \left(\frac{\varepsilon_{n}}{\sqrt{\tau}}, 1\right)
$$

which again gives a contribution to $(3.78)\left(l_{n}(u) \geqq\left((c(L) n)^{-1 / 4}\right.\right.$ for $u \leqq 2 \varepsilon_{n}$, so we take 1 as an upper bound for $\tau \leqq L^{\frac{-n}{2}}$ and $\frac{\varepsilon_{n}}{\sqrt{\tau}}$ otherwise).

The proof of (3.79) is similar to the one of (3.56), using (3.105). The proof of (3.80) follows the one of (3.58). To bound $L^{3 n} \int_{0}^{\tau}\left|V^{\prime}\left(L^{n} \omega(s)\right)\right| d s$, we use $L^{2 n}\left|V^{\prime}\left(L^{n} \omega(s)\right)\right|$ $\leqq W_{n}(\omega(s))$ for $\omega(s)<0$, where $W_{n}$ occurs in $(37,38)$. Indeed, the only property of $\bar{W}_{n}$ that we used was (38), which holds for $V^{\prime}$. Then, we use

$$
L^{3 n} \int_{0}^{\tau}\left|V^{\prime}\left(L^{n} \omega(s)\right)\right| d s \leqq L^{n} c(\lambda) e^{\frac{\lambda}{2} \int_{0}^{t_{1}} W_{n}(\omega(s)) d s} e^{\frac{1}{2} \int_{t_{1}}^{\tau} V_{n}(\omega(s)) d s},
$$


where $t_{1}$ is the first time that $\omega$ visits 0 , as in (21), and we use (3.105) for $s \geqq t_{1}$. Then we can repeat the proof of (3.58) and use $c(\lambda) L^{n} \leqq L^{n\left(1+\frac{\delta}{4}\right)}$ to obtain (3.80).

The proof of (3.81) follows the one of (3.57): consider (12); when $\omega<-\frac{\varepsilon_{n}}{2}$, we have

$$
\int_{0}^{\tau}\left|V^{\prime}\left(L^{n} \omega(s)\right)\right| d s \leqq L^{-n p}
$$

and we can get the exponential decay as in the proof of (3.78). On the other hand, the second term in (12) led to the second term in (3.57) which, after integration over $v$ contributes to the RHS of (3.81).

Proof of Lemma 5. Part a is proven just like in Lemma 3, with the only change that $f_{n}(u)$ is replaced by $-L^{n} u$.

The proof of (3.100) is similar to the one of (3.57), but for $\omega<-\frac{\varepsilon_{n}}{2}$, we have of course

$$
3 \int_{0}^{\tau} V_{n}(\omega(s)) d s \geqq 3 \tau L^{2 n}-c
$$

instead of (14). So we can take $c$ in (3.100) equal to 2 or to $\alpha-1$, if 3 in (3.25) is replaced by $\alpha$.

Finally, for (3.101) we can follow the proof of (3.58).

Acknowledgments. During the course of this work, J.B. has benefitted from the hospitality of the Mathematics Dept. of Rutgers University, and of the University of Helsinki. A.K. benefitted from the hospitality of the University of Louvain. This work was supported, in part, by the EC Grant SC1-CT91-0695 and the NSF Grant DMS-8903041.

\section{References}

1. Aronson, D.G., Weinberger, H.F.: Multidimensional non-linear diffusion arising in population genetics. Adv. Math. 30, 33-76 (1978)

2. Ben-Jacob, E., Brand, H., Dee, G., Kramers, L., Langer, J.S.: Pattern propagation in nonlinear dissipative systems. Physica 14D, 348-364 (1985)

3. Barenblatt, G.I.: Similarity, Self-Similarity and Intermediate Asymptotics. New York: Consultants Bureau 1979

4. Bramson, M.: Convergence of solutions of the Kolmogorov equation to traveling waves. Mem. Am. Math. Soc. 44, nr. 285, 1-190 (1983)

5. Bricmont, J., Kupiainen, A., Lin, G.: Renormalization Group and asymptotics of solutions of nonlinear parabolic equations. Commun. Pure Appl. Math., to appear

6. Bricmont, J., Kupiainen, A.: The Renormalization Group and the Ginzburg-Landau equation. Commun. Math. Phys., 150, 193-208 (1992)

7. Collet, P., Eckmann, J.-P.: Solutions without phase-slip for the Ginzburg-Landau equation. Commun. Math. Phys. 145, 345-356 (1992)

8. Collet, P., Eckmann, J.-P.: Space-time behaviour in problems of hydrodynamic type: A case study. Nonlinearity 5, 1265-1302 (1992)

9. Dee, G., Langer, J.S.: Propagating Pattern Selection. Phys. Rev. Lett. 50, 383 (1983)

10. Dee, G.: Dynamical properties of propagating front solutions of the amplitude equation. Physica 15D, 295-304 (1985) 
11. Fortuin, C., Kasteleyn, P., Ginibre, J.: Correlation inequalities on some partially ordered sets. Commun. Math. Phys. 22, 89-103 (1971)

12. Goldenfeld, N., Martin, O., Oono, Y., Liu, F.: Anomalous dimensions and the renormalization group in a nonlinear diffusion process. Phys. Rev. Lett. 64, 1361-1364 (1990)

13. Goldenfeld, N., Martin, O., Oono, Y.: Asymptotics of partial differential equations and the renormalization group. To appear in the Proc. of the NATO ARW on Asymptotics beyond all orders, edited by S. Tanveer (Plenum Press)

14. Simon, B.: Functional Integration and Quantum Physics. New York: Academic Press, 1979

Communicated by J.-P. Eckmann 